\title{
Evolution of the eukaryotic dynactin complex, the activator of cytoplasmic dynein
}

\author{
Björn Hammesfahr and Martin Kollmar
}

\begin{abstract}
Background: Dynactin is a large multisubunit protein complex that enhances the processivity of cytoplasmic dynein and acts as an adapter between dynein and the cargo. It is composed of eleven different polypeptides of which eight are unique to this complex, namely dynactin1 ( $150^{\text {Glued }}$ ), dynactin2 (p50 or dynamitin), dynactin3 (p24), dynactin4 (p62), dynactin5 (p25), dynactin6 (p27), and the actin-related proteins Arp1 and Arp10 (Arp11).

Results: To reveal the evolution of dynactin across the eukaryotic tree the presence or absence of all dynactin subunits was determined in most of the available eukaryotic genome assemblies. Altogether, 3061 dynactin sequences from 478 organisms have been annotated. Phylogenetic trees of the various subunit sequences were used to reveal sub-family relationships and to reconstruct gene duplication events. Especially in the metazoan lineage, several of the dynactin subunits were duplicated independently in different branches. The largest subunit repertoire is found in vertebrates. Dynactin diversity in vertebrates is further increased by alternative splicing of several subunits. The most prominent example is the dynactin 1 gene, which may code for up to 36 different isoforms due to three different transcription start sites and four exons that are spliced as differentially included exons.

Conclusions: The dynactin complex is a very ancient complex that most likely included all subunits in the last common ancestor of extant eukaryotes. The absence of dynactin in certain species coincides with that of the cytoplasmic dynein heavy chain: Organisms that do not encode cytoplasmic dynein like plants and diplomonads also do not encode the unique dynactin subunits. The conserved core of dynactin consists of dynactin1, dynactin2, dynactin4, dynactin5, Arp1, and the heterodimeric actin capping protein. The evolution of the remaining subunits dynactin3, dynactin6, and Arp10 is characterized by many branch- and species-specific gene loss events.
\end{abstract}

\section{Background}

Dynactin is a multisubunit protein complex in eukaryotic cells required as an activator of cytoplasmic dynein, the major minus end-directed microtubule motor [1,2]. Dynactin acts as an adapter between dynein and the cargo [2-4] and enhances the movement of dynein by increasing its processivity [5-8]. The dynein-dynactin complex plays an important role during mitosis $[9,10]$ and is necessary for synapse stabilization [11]. It is involved in nuclear migration, and during cell division in mitotic spindle positioning [12-14] and organization of spindle microtubule arrays [15]. Although most of dynactins functions are in conjunction with cytoplasmic dynein it also binds to and modulates kinesin-2 [16,17] and kinesin-5 [18].

\footnotetext{
* Correspondence: mako@nmr.mpibpc.mpg.de

Abteilung NMR basierte Strukturbiologie, Max-Planck-Institut für Biophysikalische Chemie, Am Fassberg 11, Göttingen D-37077, Germany
}

Dynactin is composed of eleven different subunits ranging in size from 22 to $150 \mathrm{kDa}$ [19]. Several components are present as dimers or oligomers in the complex resulting in an overall molecular weight of $1.2 \mathrm{MDa}$. The novel dynactin subunits have initially been named according to the molecular weights of the vertebrate subunits in SDS gels [1]. However, as the molecular weights differ between species the original naming is not adequate to describe the protein family relation of the subunits in all eukaryotes. Therefore and because these subunits are unique to the dynactin complex we adopt and use the nomenclature dynactin1 to dynactin6 (symbols DCTN1 to DCTN6), which has recently been established by the HUGO Gene Nomenclature Committee (HGNC; [20]), throughout this analysis.

The structure of the complex can be divided into two distinct domains: the Arp1 rod and the projecting arm $[21,22]$. The projecting arm (consisting of the so-called
C Biomed Central 
sidearm and shoulder complex) links dynactin to cytoplasmic dynein, kinesin motors, and microtubules. It is composed of two dynactin1 ( $150^{\text {Glued }}$ ), four dynactin2 (p50 or dynamitin), and two dynactin3 subunits (p24 and p22 have been used for the mouse and the human ortholog, respectively). The Arp1 rod is built of eight Arp1 molecules forming a short actin-like filament, probably one $\beta$-actin molecule, and the conventional actin capping proteins Cap $\alpha$ and $C a p \beta$, which are located at the barbed-end of the mini-filament. The other end of the filament is terminated by Arp10 (the name Arp11 is synonymously used for the vertebrate orthologs [20]) and dynactin4 (p62), to which the dynactin5 (p25) and dynactin6 (p27) subunits are associated. The heterotetrameric complex of dynactin4, dynactin5, dynactin 6 and Arp10 is also called pointed-end complex.

Dynactin1 is the largest subunit of the dynactin complex [23] and belongs to the microtubule plus endbinding protein family [24]. The microtubule-binding CAP-Gly (cytoskeleton-associated protein-glycine-rich) domain is located at the N-terminus [23,25]. The CAPGly domain is connected to the other subunits of the complex via two long coiled-coil regions. The first coiled-coil region following the CAP-Gly domain binds to the intermediate chain of cytoplasmic dynein [26,27]. Dynactin2 is the connection between the projecting arm and the Arp1 rod [28,29] and its over-expression in vivo causes disruption of the dynactin complex [21,28,30]. Dynactin3 is required for attachment of dynactin1 to dynactin2 [31]. Arp1 is the actin-related protein most similar to actin and forms an actin-like mini-filament [22] that represents the backbone of dynactin, to which the other dynactin subunits bind. It is supposed that membranous cargoes bind to dynactin via the Arp1 rod $[4,32,33]$.

The first studies on dynactin have been performed with chicken brain samples [1,2]. Subsequently, dynactin subunits have been identified and analyzed in the model organisms Neurospora crassa [34-39], Saccharomyces cerevisiae [40-43], Drosophila melanogaster [44,45] and Caenorhabditis elegans [45-47]. Although the composition of the dynactin complex in vertebrates gradually became apparent, a thorough analysis of the complex and its subunits in terms of gene duplicates, alternatively spliced isoforms, and phylogenetic evolution is still missing. That a surprising diversity might be found has been shown by a recent study of the motor protein repertoire of 21 insect genomes uncovering a branch specific duplication of the well-known dynactin1 ( $\left.150^{\text {Glued }}\right)$ gene in Drosophila species [48].

Building such a multi-protein complex with a filament of fixed size seems rather complicated. Because most of the analyses of the complex have been done with vertebrate samples, it would be interesting to see whether the various unicellular protists that often have smaller gene repertoires, may have evolved compacted versions of the dynactin complex. Vice-versa, there could have been a minimal dynactin complex at the origin of the eukaryotes that multicellular eukaryotes expanded to accomplish more and different tasks. Here, we examined every known protein of the complex and determined its absence and presence in all eukaryotic genomes as available in September 2011. Furthermore, we inspected all genes to identify alternatively spliced exons and their appearance during evolution. For our analysis, we manually assembled and annotated more than 4,700 dynactin and actin-related protein sequences from about 550 species. All sequences were inspected and validated at the genomic DNA level to remove wrongly predicted sequence regions, to manually fill gaps in gene predictions, and to reveal the correct exon/intron boundaries. The sequences and related data like gene structure reconstructions and biochemical properties are available through CyMoBase (http://www.cymobase.org).

\section{Results}

\section{Identification of dynactin genes}

Dynactin protein sequences are not as strongly conserved as for example tubulins, and three of the dynactin subunits are relatively short complicating their identification if they were spread on several exons. In addition, dynactin contains two actin-related proteins of which Arp1 is closely related to actin while Arp10 is a very divergent member thus hindering their immediate identification. The dynactin subunits might have been duplicated in single species or certain branches, like the Drosophila dynactin1 gene [48]. These events can only be revealed through the phylogenetic analysis of the corresponding protein sequences. Thus, it is of major importance to obtain the best sequence data possible and to create the most accurate multiple sequence alignments. Automatic gene predictions are error-prone (for example, automatic gene prediction programs do not recognize GC---AG intron splice sites), and even those gene predictions are available for only a small subset of all sequenced eukaryotic genomes [49]. Therefore, we manually assembled and annotated all dynactin and actin-related sequences used in this study. Manual identification and assembly means that we started from a set of sequences verified by cDNA and used those for searches with standard tools like TBLASTN in the genome assemblies. Unfortunately, only a few full-length mRNA/cDNA sequences for dynactin subunits are available, which served as representatives for correct sequences. Every search hit has further been analysed by manual inspection of the corresponding genomic DNA sequence either to reveal the correct intron/exon boundaries or to extend hits that only covered short parts of 
the search sequence. Those sequences were excluded, for which some local similarity was identified (e.g. similarity to the dynactin1 CAP-Gly domain) but for which the remaining parts of the respective subunits could not be found although the genomic sequences of the respective contigs were long enough. Genomes, for which the respective dynactin subunits could not unambiguously be assembled in the first instance, were reanalysed as soon as further data was added to the multiple sequence alignments. In this way the completeness of the search for dynactin subunits and the accuracy of the gene assembly and annotation has continuously been reevaluated and improved. In addition to manually assembling all sequences, the multiple sequence alignments of the dynactin sequences have been created and were maintained and improved manually (Additional file 1).

Sequences of which small parts were missing due to gaps in the genome assemblies (up to 5\%) were termed "Partials". "Partials" are not expected to considerably influence the phylogenetic tree computations. Sequences of which more than $5 \%$ were missing due to genome assembly gaps or incomplete EST data but that are otherwise unambiguous orthologs or paralogs were termed "Fragments". "Fragments" are important to denote the presence of the subunits in the respective species in the qualitative analysis. Dynactin genes were termed pseudogenes if they contain more features like frame shifts and in-frame stop codons and miss more conserved sequence regions than can be attributed to sequencing or assembly errors.

In total, the dynactin dataset contains 3061 sequences from 478 organisms (Table 1, Additional file 2), of which 2872 have been derived from 353 WGS sequencing projects. 2668 sequences are complete, and an additional 191 sequences are partially complete. In addition, 1766 actin and actinrelated proteins from 323 species have been assembled to finally reveal the subfamily relationship of potential Arp1 and Arp10 orthologs in questionable cases. For plotting the presence or absence of dynactin subunits across the tree of the eukaryotes we only included those species whose genomes have been sequenced with high coverage and which provided reliable data in many other cases [48,50-52]. Nevertheless, low-coverage genomes have also been analysed because every single piece of sequence could be very important to resolve ambiguous regions in related species or to clarify phylogenetic question. For example, we also analysed the incomplete genome of the agnath Petromyzon marinus to reveal at which stage alternative splice forms had been evolved in vertebrate evolution. To infer the phylogenetic relationship of duplicated dynactin subunits we calculated phylogenetic trees using the Maximum Likelihood and
Bayesian methods. Gene structures were reconstructed for all sequences using WebScipio [53] and can be inspected via CyMoBase (www.cymobase.org) for further investigation.

\section{Dynactin 1}

Dynactin1 plays a major role for the function of the dynactin complex as it connects the Arp1 rod, and thus the cargo binding sites, to cytoplasmic dynein, the transporter protein complex, and to microtubules, the track. It can hardly be imagined to build a functional dynactin complex without a dynactin1 subunit. However, dynactin1 is also the least conserved of the dynactin subunits (Figure 1). This is most likely due to its domain structure that consists of a short $\mathrm{N}$-terminal globular CAP-Gly domain followed by two coiled-coil regions, which account for two thirds of its primary sequence. Both the region separating the two coiled-coil regions and the $\mathrm{C}$-terminal region are not even conserved between metazoan and fungal dynactin1 subunits, which belong to the opisthokont branch. Given the functional importance of dynactin1 we were surprised not to be able to identify homologs in any Apicomplexa, in the Heterolobosea Naegleria gruberi, and the Apusozoa Thecamonas trahens (Table 1). When searching for dynactin1 homologs in these organisms we analysed all TBLASTN and PSI-BLAST hits showing sequence similarity to CAP-Gly domains but we only found other CAP-Gly domain containing proteins like CLIP-170/restin [54], and the tubulin-specific chaperones B and E $[55,56]$.

Duplicates of dynactin1 have been found in independent branches of the eukaryotic tree (Additional file 3). In the Brachycera branch (including the Drosophila clade) the dynactin1 gene has been duplicated once [48]. Another duplication of dynactin1 was found in the Actinopterygii branch, supported by Brachydanio rerio, Takifugu rubripes, and Gasterosteus aculeatus. Some of the nematods like Brugia malayi also encode two versions of dynactin1. Two duplications of dynactin 1 were found in the genome of the fungus Rhizopus arrhizus, and one additional dynactin1 in Mucor circinelloides. The variant A and B subunits each grouped together, suggesting a gene duplication predating the separation of the two species. Variant C of Rhizopus arrhizus grouped to variant B indicating another Rhizopusspecific duplication.

The dynactin1 gene of Homo sapiens is encoded in 32 exons on chromosome 2 (Figure 2A, [57]). All exons are constitutively expressed and present in all dynactin 1 transcripts, except for exon 5 ("RGLKPKK"), the second part of exon 6 ("APTARK"), exon 7 ("TTTRRPK"), and exon 27 ("EEQQR") that are alternatively spliced (Figure 2B). Some alternative transcripts have already been described based on the analysis of a fetal human cDNA library (dynactin1- $\Delta 5$; dynactin1- $\Delta 5,6$ : dynactin $1-\Delta 5,6,7 ;$ [58]) suggesting that 
Table 1 Data statistics

\begin{tabular}{|c|c|c|c|c|c|c|c|c|c|c|c|}
\hline & Total & $\begin{array}{l}\text { Dynactin1 } \\
\text { p150 } \\
\text { ropy-3 } \\
\text { Nip100 }\end{array}$ & $\begin{array}{l}\text { Dynactin2 } \\
\text { p50 } \\
\text { Jnm1 }\end{array}$ & $\begin{array}{l}\text { Dynactin3 } \\
\text { p24 } \\
\text { ropy-10 } \\
\text { Ldb18 }\end{array}$ & $\begin{array}{l}\text { Dynactin4 } \\
\text { p62 } \\
\text { ropy-2 }\end{array}$ & $\begin{array}{l}\text { Dynactin5 } \\
\text { p25 } \\
\text { ropy-12 }\end{array}$ & $\begin{array}{l}\text { Dynactin6 } \\
\text { p27 }\end{array}$ & $\begin{array}{l}\text { Cap1 } \\
\text { (Capa) }\end{array}$ & $\begin{array}{l}\text { Cap2 } \\
(\text { Cap } \beta)\end{array}$ & $\begin{array}{l}\text { Arp1 } \\
\text { ropy-4 }\end{array}$ & $\begin{array}{l}\text { Arp10 } \\
\text { Arp11 } \\
\text { ropy-7 }\end{array}$ \\
\hline \multicolumn{12}{|l|}{ Sequence } \\
\hline Total & 3061 & 321 & 312 & 278 & 322 & 326 & 258 & 368 & 299 & 306 & 271 \\
\hline From WGS & 2872 & 300 & 280 & 248 & 289 & 296 & 229 & 359 & 298 & 304 & 269 \\
\hline Pseudogenes & 60 & 1 & 6 & 3 & 19 & 12 & 5 & 14 & 0 & 0 & 0 \\
\hline \multicolumn{12}{|l|}{ Completeness } \\
\hline Complete & 2668 & 246 & 259 & 250 & 250 & 273 & 220 & 345 & 286 & 293 & 246 \\
\hline Partials & 191 & 27 & 14 & 23 & 28 & 31 & 27 & 10 & 4 & 10 & 17 \\
\hline Fragments & 181 & 48 & 37 & 5 & 44 & 12 & 9 & 6 & 9 & 3 & 8 \\
\hline \multicolumn{12}{|l|}{ Species } \\
\hline Total & 2863 & 288 & 306 & 274 & 301 & 313 & 246 & 289 & 292 & 287 & 267 \\
\hline WGS-projects & 2567 & 257 & 261 & 238 & 256 & 271 & 215 & 270 & 278 & 269 & 252 \\
\hline EST-projects & 960 & 86 & 106 & 96 & 103 & 113 & 93 & 102 & 102 & 87 & 72 \\
\hline $\begin{array}{l}\text { WGS- and } \\
\text { EST-projects }\end{array}$ & 1314 & 124 & 135 & 116 & 134 & 148 & 115 & 146 & 149 & 133 & 114 \\
\hline \multicolumn{12}{|l|}{$\begin{array}{l}\text { Sequences } \\
\text { in Taxa }\end{array}$} \\
\hline Metazoa & 1339 & 155 & 132 & 141 & 159 & 158 & 132 & 167 & 94 & 101 & 100 \\
\hline Fungi & 1339 & 144 & 140 & 118 & 124 & 118 & 96 & 138 & 141 & 165 & 155 \\
\hline Apusozoa & 9 & 0 & 1 & 1 & 1 & 1 & 1 & 1 & 1 & 1 & 1 \\
\hline Amoeba & 56 & 5 & 5 & 4 & 5 & 5 & 5 & 10 & 8 & 4 & 5 \\
\hline SAR & 227 & 15 & 28 & 10 & 27 & 32 & 17 & 30 & 29 & 30 & 9 \\
\hline Excavata & 21 & 0 & 1 & 0 & 1 & 5 & 2 & 4 & 7 & 1 & 0 \\
\hline Viridiplantae & 53 & 0 & 0 & 0 & 0 & 0 & 0 & 27 & 26 & 0 & 0 \\
\hline Rhodophyta & 0 & 0 & 0 & 0 & 0 & 0 & 0 & 0 & 0 & 0 & 0 \\
\hline Glaucophyta & 5 & 0 & 1 & 1 & 1 & 2 & 0 & 1 & 1 & 0 & 0 \\
\hline Cryptophyta & 3 & 0 & 1 & 0 & 0 & 1 & 1 & 0 & 0 & 0 & 0 \\
\hline Haptophyta & 0 & 0 & 0 & 0 & 0 & 0 & 0 & 0 & 0 & 0 & 0 \\
\hline
\end{tabular}

exons 5-7 are each differentially included. In order to reveal a more general view of possible transcripts we extensively searched for corresponding sequences of vertebrate species in the available EST and cDNA databases and found the following combinations for exons 5-7 (Figure 2C):

- none of the alternative exons is included in the transcript $(\Delta 5,6,7)$

- exon 5 included, resulting in four additional positively charged residues (lysines or arginines, $\Delta 6,7)$

- exon 7 included, three additional positively charged residues $(\Delta 5,6)$

- exon 5 and 7 included, seven additional positively charged residues $(\Delta 6)$

- exon 6 and 7 included, five additional positively charged residues $(\Delta 5)$
- exon 5, 6 und 7 included, nine additional positively charged residues

We did not find EST or cDNA-data for transcripts including only exon $6(\Delta 5,7)$, or EST-data including exons 5 and 6 without exon $7(\Delta 7)$. Exon 27 is also a differentially included exon. Maybe because of lack of more fulllength cDNA data or maybe because of tight regulation, exon 27 is found to be absent in dynactin $1-\Delta 5,6,7$, and to be present in dynactin1- $\Delta 5$ and dynactin1 (Figure 2C). In addition, transcripts are generated from three alternative start positions. The first is at the beginning of exon 1, the second is at the beginning of exon 2, and the third possible transcript starts with exon 6 ("MMRQAPTARK..."), which corresponds to the "p135" construct. While transcript start sites 1 and 2 are found in all described combinations of exons 5-7 

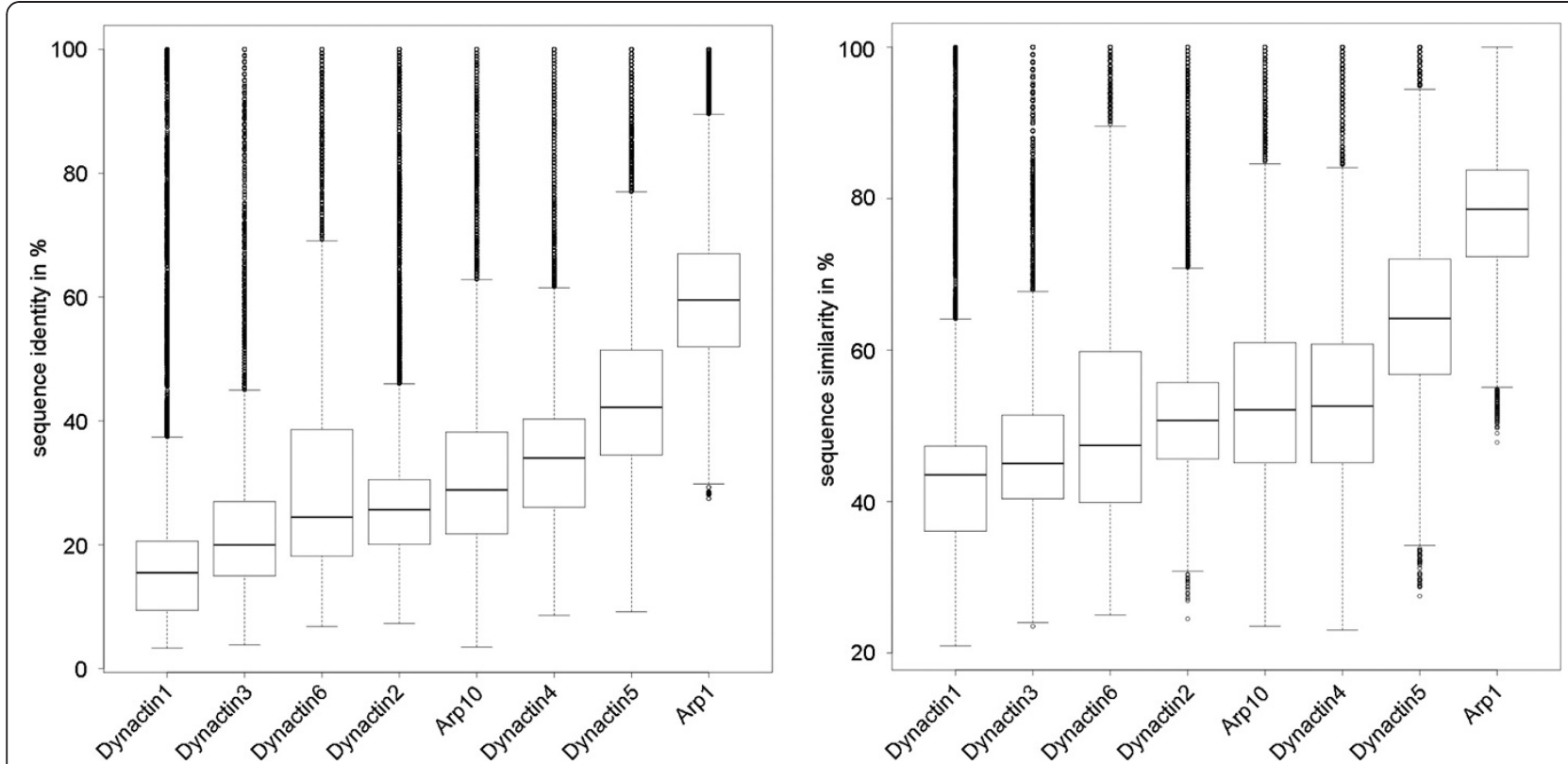

Figure 1 Sequence conservation in dynactin subunits. Box plots of the sequence identities and similarities of the dynactin subunits.

and exon 27, transcript start site 3 (exon 6) is only found in combination with exon 7 included and either exon 27 included or spliced out.

Interestingly, the alternative exons encode different numbers of basic residues, arginines and lysines. Although only six of the eight possible combinations of the alternative exons have been found in EST and cDNA data so far, vertebrates seem to be able to stepwise increase the number of basic residues in this region from zero to nine. The basic residues influence the sliding behaviour of dynactin along the microtubules with fewer charges allowing a faster diffusion [58]. The function of the region including the fourth differentially included exon, exon 27, which is located subsequent to the second coiled-coil region and thus behind a proposed Arp1 binding site [23], has not been analysed so far. While the third transcription start site produces a dynactin 1 without a CAP-Gly domain ("p135") the functional difference between transcripts of the two other transcription start sites is not known yet. The longer N-terminus (about 20 residues) is not visible in any of the available crystal structures of dynactin1 CAP-Gly domains [25,59-61]. In addition, a solution state structure (PDBid 2COY) revealed that the $\mathrm{N}$-terminus is an unstructured and unordered coil.

There is another alternative transcript generated by retention of intron 22 (Figure 2A). This intron retention results in a premature stop codon and has only been found in combination with transcription start site 2 . The resulting transcript includes the CAP-Gly microtubule binding domain and the dynein intermediate chain binding site but stops before the second proposed coiled-coil region. The C-terminal part of dynactin1 starting with the second coiled-coil region has been proposed to bind to Arp1 and truncation mutants of Drosophila dynactin 1 have been shown not to be incorporated into dynactin $[23,62]$. This most likely also accounts for the alternative transcripts including intron 22 of vertebrate dynactin 1.

The alternatively spliced exons and transcription start sites are conserved in all vertebrates and were also found in the agnath Petromyzon marinus the sistergroup of all gnathostomes representing the deepest separation in extant vertebrates. Especially the lysines and arginines and their positions are invariant. However, in the fish type A dynactin 1 subunits the exons 5 have been lost, as well as the third potential translation start in exon 6. Instead, exon 6 encodes only the part that is alternatively spliced in type B dynactin1. Thus a "p135"-like isoform cannot be build from fish type A dynactin1 subunits. Alternatively spliced isoforms have not been identified in any other of the analysed species.

The sequence conservation plot across all dynactin 1 CAP-Gly domains shows that the core structure consisting of six beta-strands and several key residues for binding microtubule plus end-tracking proteins is highly conserved (Figure 2D). The key residues for binding the C-terminal EEY/F tail motifs of CLIP170, EB1 proteins, and $\alpha$-tubulines are F52, W57, K68, N69, and R90 (human dynactin1 numbering, [25,59]). These are almost invariant from stramenopiles to alveolates to humans (Figure 2D). In contrast, the residues of the proposed second EB1-binding site A49, L51, T54, K56, and R76 (human dynactin1 numbering, [59]) are not conserved (Figure 2D). EB1 proteins are present in all eukaryotes 


\section{A dynactin1 Hs p150}

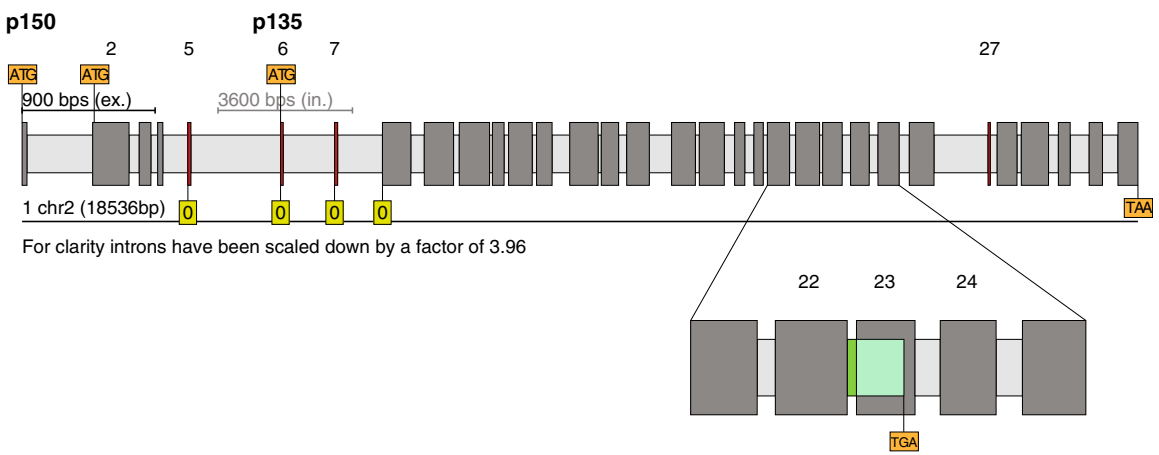

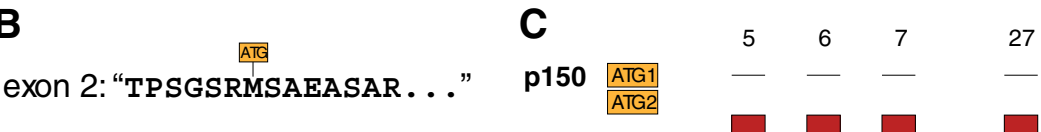

$$
\begin{aligned}
& \text { exon 5: "RGLKPKR" }
\end{aligned}
$$

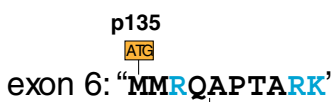

$$
\begin{aligned}
& \begin{array}{r}
\text { exon 6. MMR } \\
0
\end{array} \\
& \square--\mathrm{ND} \\
& \square-\square \quad \text { ND } \\
& \text { eXon 7: "TTTRRPK" } \\
& \text { exon 27: "EEQQR" } \\
& \text { p135 ATG3 }
\end{aligned}
$$

D

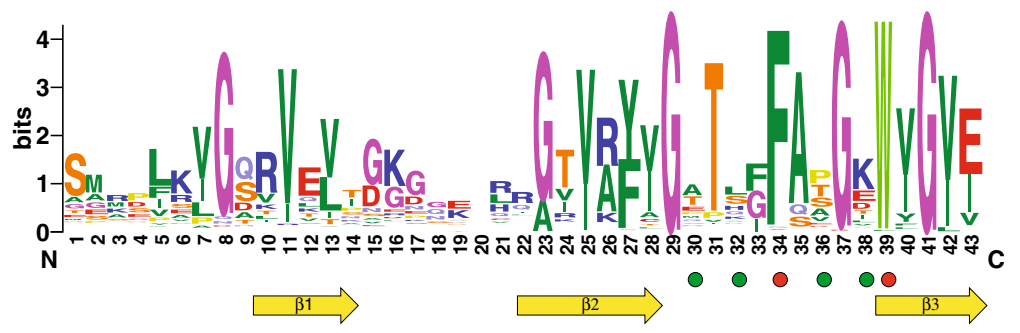

SC 11 NMQNISLQDTVLVNEMK- - - - GRVKFIGETQ- FAKGIWYGIE

Hs 23 SARPLRVGSRVEVIGKG- - - HRGTAYVGATL-FATGKWVGVI

Dd 4 EGKSLPPGTRVINGKP-ELGLGVKYCGMTK-FSPGRWVGE

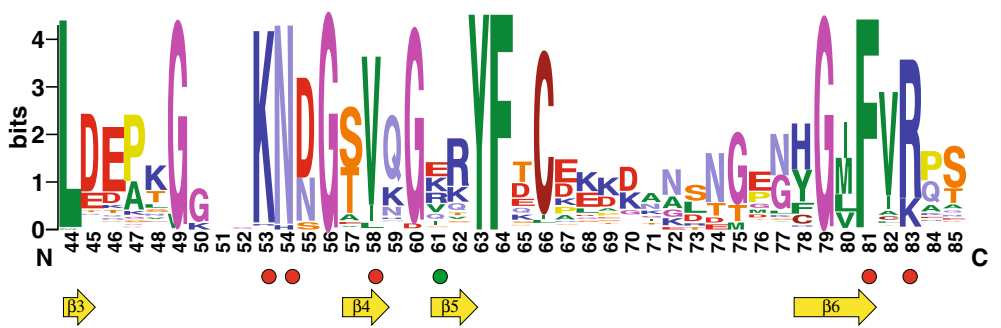

SC 48 LDKPLG- - - KNDGSVNGIRYFDIDLKKANSNGGYYGLFCKRD 86

Hs 62 LDEAKG - - KNDGTVQGRKYFTCD - . - . - - EGHGIFVRQS 92

Dd 45 LDSAAG - - KNDGTVQGERYFDCK - . - - - PAHGLFVKPN 75

$G^{71} A / R / E Q^{72} P$

Figure 2 (See legend on next page.) 
(See figure on previous page.)

Figure 2 Gene structure and isoforms generated by alternative splicing of dynactin1. The gene structure was reconstructed with Webscipio and represents the dynactin1 (p150) homolog of Homo sapiens encoded by 32 exons including four alternatively spliced exons (A). Dark gray bars represent exons, light gray bars indicate introns, and coloured bars symbolize the alternatively spliced exons. For better visualisation, exons and introns are scaled differently. ATG in orange rectangles represent translation start positions. Translation start codons exist in exons 1, 2, and 6, respectively. The zero in green rectangles represents the first reading frame. A zoomed view on the exons $21-25$ shows intron retention of intron 22 (dark-green bar) that results in the translation of exon 23 in a different reading frame leading to a premature stop codon (light-green bar). The protein sequences for the alternative exons are given (B) as well as a short summary of the combinations of the alternatively spliced exons that have been found in full-length cDNA data (C). Due to missing full-length cDNA sequences the inclusion or exclusion of alternative exon 27 could not be determined for all combinations of exons 5 to 7 (ND=not determined). The sequence logos (D) illustrate the sequence conservation within the multiple sequence alignment of the CAP-Gly domain. For better orientation, the sequences of three representative CAP-Gly domains are shown: the human CAP-Gly domain as the main target of disease associated mutations, the Saccharomyces cerevisiae and the Dictyostelium CAP-Gly domains as representatives of widely used model organisms. $\beta$-strands as determined from the crystal structure are drawn as yellow arrows. Green dots point to amino acids of the human CAP-Gly domain that have been proposed to constitute the second EB1-binding site [59] and red dots highlight residues that are part of the conserved EEY/F motif binding site [25,59,60]. Some mutations as found in human diseases are given below the reference sequences with numbering referring to human dynactin 1 .

(plants, Giardia, stramenopiles, Alveolata, Trichomonas, Opisthokonts, data not shown). Thus, this proposed second EB1-binding site could be specific to mammals or, most likely, be an artefact from crystal packing effects. The latter is supported by another crystal structure of the complex of the dynactin CAP-Gly domain and the C-terminus of EB1, in which only the C-terminal EEY motif binds to dynactin1 [60]. Several mutations in the CAP-Gly domain of human dynactin 1 are associated with diseases. The G59S mutation has been identified in patients with distal spinal bulbar muscular atrophy (dSBMA, [63]) and the G71R/E/A, T72P, and Q74P mutations have been found in patients with Perry's syndrome [64]. All mutations lead to destabilization of the CAP-Gly domain [65]. The two glycines G59 and G71 are invariant in all dynactin1 CAP-Gly domains. While the threonine and glutamine are variable across the eukaryotes prolines are never found at these positions (Figure 2D).

\section{Dynactin2}

Dynactin2 was found in almost all branches of the eukaryotic tree that contain a dynactin complex (Table 1). The only two species containing a likely functional dynactin complex without dynactin2 are the closely related yeasts Ogataea parapolymorpha and Ogataea angusta. Because two different species of Ogataea have been sequenced it is unlikely that dynactin 2 could be missed because of gaps in the assemblies. None of the genomes analysed encodes more than one functional dynactin2 gene. Some mammals and Caenorhabditis brenneri contain dynactin 2 pseudogenes.

Dynactin2 from Homo sapiens is encoded in 16 exons on chromosome 12 (Figure 3A). Two of the exons, the very short exons exon 3 ("FAQ", residues 36-38) and exon 4 ("EL", residues 39 and 40), are alternatively spliced. Both exons are independently differentially included and many EST and cDNA clones from many vertebrates exist excluding exons 3 and 4 (dynactin2- $\Delta 3$,
4) as well as including each exon separately (dynactin2$\Delta 3$ and dynactin2- $\Delta 4$ ) and both exons together. The two alternatively spliced exons were also found in the agnath Petromyzon marinus, but not in any invertebrate and thus seem to be an invention of the most ancient vertebrate. While the up- and downstream coding sequence around exons 3 and 4 is slightly variable in vertebrates, the sequence of the two short exons is invariant. In contrast to dynactin 1 we could not identify any further transcription start sites. The analysis of the available EST/ cDNA data do not support alternatively spliced isoforms in any other species than vertebrates.

The first dynactin2 cDNA sequences were isolated from rat and human, and consisted of the long form including both alternative exons (isoform-1, [28]). Although immunobiochemical studies of the dynactin 2 expression in various adult rat tissues have been interpreted to result from the same transcript [28] the slightly different sizes of the dynactin 2 bands in the SDS-gels could in retrospect originate from the tissue-specific expression of the alternative splice forms. Later, isoform-1 and the dynactin2 isoform excluding the two alternative exons (isoform-2, dynactin2$\Delta 3,4)$ have been shown to be tissue specific transcribed [66], and very recently isoform-2 from human has been compared to chicken dynactin2- $\Delta 3$ with respect to determinants for self-oligomerization and interactions with other dynactin subunits [29].

The residues encoded by the alternative exons (residues 36 to 40 ) are located in the N-terminal region of dynactin2 but have not been the specific focus of any biochemical study yet. Both the $\mathrm{N}$-terminal and the C-terminal part of dynactin 2 are needed for proper self-assembly and binding to dynactin3. The $\mathrm{N}$-terminal 100 residues seem to be required and sufficient for binding to Arp1 $[29,67]$. Binding essays showed that determinants for the optimal recruitment of dynactin 1 are located in the $\mathrm{N}$-terminal half of dynactin2 but that the $\mathrm{N}$-terminal 100 residues alone are not sufficient [29]. It could thus be possible that 


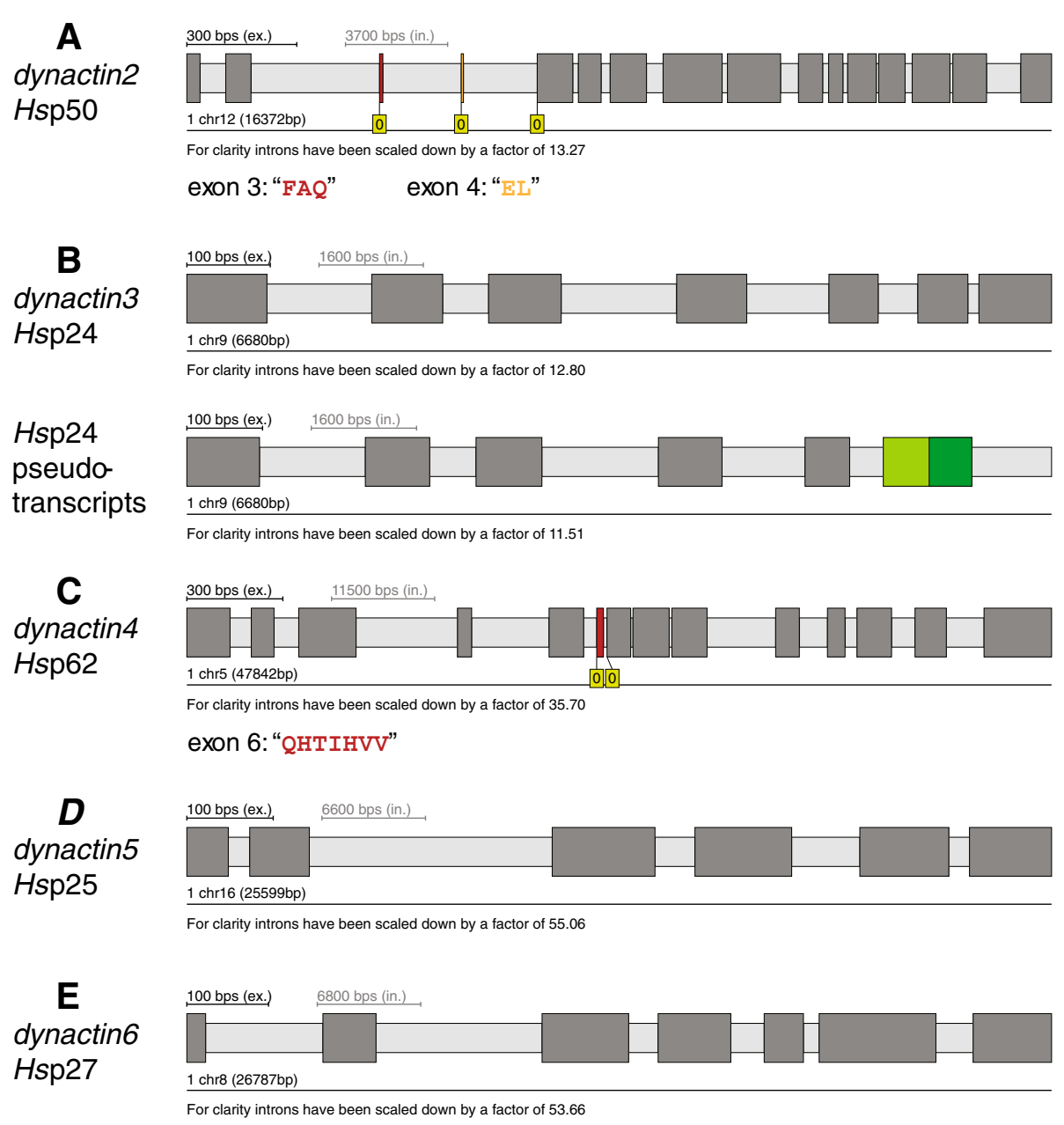

Figure 3 Gene structures and alternatively spliced exons of dynactin subunits. The gene structures including alternatively spliced exons of the dynactin subunits of Homo sapiens were reconstructed with WebScipio. The colour coding is the same as in Figure 2 A) The scheme shows the gene structure of dynactin2 (p50) consisting of 16 exons including the differentially included exons 3 and 4. B) Gene structure of dynactin3 (7 exons). For dynactin3, pseudo-transcripts were identified (for detailed information see Additional File 4). C) The dynactin4 (p62) gene is comprised of 14 exons of which exon 6 is alternatively spliced. E) and F) Gene structures of dynactin5 (6 exons) and dynactin6 (7 exons), respectively.

a certain combination of alternatively spliced exons in dynactin2 correlates with the differentially inclusion of exon 27 of dynactin1. More specific experiments will be necessary to reveal how such small modifications of two to five residues could modify dynactin2's binding to Arp1, dynactin1, and dynactin3.

\section{Dynactin3}

We were not able to identify dynactin3 homologs in Ustilaginomycetes, Chytridiomycota, Naegleria gruberi, Bigelowiella natans, Ciliophora, plants, and Stramenopiles (Table 1). Dynactin3 homologs could also not be identified in the Schizosaccharomyces branch and most of the analysed yeast species. It has been proposed that dynactin 3 is the least conserved of the dynactin subunits [45]. This analysis has been based on the comparison of the sequence identities of the dynactin subunits of chicken,
Drosophila, C. elegans, and Neurospora crassa to the mouse subunits. In order to determine the least conserved dynactin subunit based on all eukaryotes we calculated sequence identity and similarity matrices for all subunits (Figure 1). Because the data includes sequences from all branches of the eukaryotes each subunit shows a broad distribution. The comparison of the medians of the populations shows that dynactin1 is the least conserved dynactin subunit followed by dynactin 3 and dynactin6. Because we were able to identify dynactin3 in almost all opisthokonts the dynactin3 subunits have most likely been lost independently in most Saccharomyctes, the Basidiomycote Ustilago maydis, and in the fungi of the Chytridiomycota. Similarly we should have been able to find the dynactin3 homologs in ciliates based on the dynactin3 subunits from the Apicomplexa. The other branches, for which we could not find dynactin3 homologs, have either lost the gene or 
the dynactin3 proteins must be very different from the known dynactin3 subunits. Naegleria, Bigelowiella, and stramenopiles species normally do not contain intron-rich genes. Thus, it is unlikely that we missed dynactin3 subunits because they were not present in gene prediction datasets (that are available for some species and that we searched with PSI-BLAST) or because the scores of short exon hits were too low to be detected with TBLASTN.

Dynactin3 has been duplicated in Rattus norvegicus. The translations of both genes are identical except for three amino acids that are conserved substitutions. However, the gene of homolog B does not contain any introns and is not supported by EST data. Therefore, it is most likely the result of a recent retro-transcription of a processed pseudogene. Human dynactin3 is encoded on chromosome 9 in 7 exons, which are constitutively spliced (Figure 3B). A few EST clones suggest the alternative transcription of exon 6 that, however, leads to pseudo-transcripts (Figure 3B, Additional file 4). Alternatively spliced isoforms have also not been identified in any other species.

\section{Dynactin4}

Dynactin4 was found in all branches of the eukaryotic tree that contain dynactin. However, homologs could not be identified in many yeast and most of the Schizosaccharomyces species. Dynactin4 proteins are much longer than dynactin3 proteins and we would expect to identify homologs in the yeast and Schizosaccharomyces species based on the supposed homology to the identified dynactin4 proteins. Missing dynactin4 genes are therefore rather the result of gene loss than the result of identification problems.

The published dynactin4 sequence from Neurospora crassa (ropy-2 or RO2 gene) contains a sequencing error that led to a predicted N-terminal extension of 173 residues [37]. The genomic sequence encodes another methionine 62 residues upstream of the translation start site. Homologous sequence to these 62 residues including the methionine could only be found in Neurospora species and the closely related Sordaria macrospora but not in other Sordariales (e.g. Chaetomium, Thielavia) or any other fungi. The sequence starting from the second methionine is highly conserved in all fungi and thus this methionine is most probably also the translation start site in Neurospora and Sordaria (Additional file 5).

Dynactin4 from Homo sapiens is encoded in 14 exons on chromosome 5 (Figure 3C). Exon 6 ("QHTIHVV") is a differentially included alternatively spliced exon. Different isoforms have already been reported for rat [68] but not further evaluated. The alternatively spliced exon is conserved in sequence, length, and reading frame in all vertebrates and was also found in the agnath Petromyzon marinus, but not in cephalochordates (Branchiostoma floridae), tunicates (e.g. Ciona intestinalis), and other invertebrates. The exon invention event therefore predates the separation of the Gnathostomata and the Hyperoartia. There is not enough EST/cDNA data available to proof the alternative character of the exon in all vertebrates. For example, there is only one EST clone from Petromyzon that covers the respective sequence region and includes exon 6 but none without exon 6. But because there are EST/cDNA clones for several fish, mammals, and Xenopus with and without exon 6 it is highly probable that exon 6 is alternatively spliced in all vertebrates. Alternatively spliced isoforms have not been identified in any other species.

Dynactin4 subunits have been predicted to contain $\mathrm{N}$ terminal LIM [68] or RING domains [69], which are short domains consisting of two zinc fingers of the treble clef fold group arranged in tandem [70,71]. The treble clef fold is characterised by a $\beta$-hairpin at the $\mathrm{N}$-terminus and an $\alpha$-helix at the $\mathrm{C}$-terminus that both contribute two ligands for zinc binding [72]. In LIM domains these ligands are almost exclusively cysteins while cysteins could be replaced by histidines in RING domains. In addition, the tandem treble clef fingers are separated by a two-residue spacer in LIM domains, which is invariant in length and seems to be essential for LIM-domain function [73]. Dynactin4 subunits from almost all species contain eight $\mathrm{CxxC}$ motifs, of which the seventh and eights motif are separated by about 150 residues. The cysteins are never substituted by histidines and a two-residue spacer exists only between the fifth and sixth motif. A multiple sequence alignment based secondary structure prediction using Jpred [74] did not reveal any $\alpha$-helical propensity close to the $\mathrm{CxxC}$ motifs (data not shown). Thus, dynactin4 can bind up to four zinc ions but it is unlikely that these zinc fingers adopt the treble clef fold and form LIM or RING domains. Rather, the CxxC motifs will form so-called zinc ribbons, which are composed of two $\beta$-hairpins forming two structurally similar zinc-binding sub-sites. These sites are often separated by even protein domains [72]. Thus, as long as structural data is not available it is not possible to predict to which of the other motifs the eighth $\mathrm{CxxC}$ motif of dynactin 4 might fold to build a zinc finger. The highly probable contribution of the eighth motif to the structure of the zinc-finger domain might also explain why expression of only the $\mathrm{N}$-terminal 130 residues of dynactin 4 resulted in aggregates [68].

\section{Dynactin5}

Dynactin 5 was found in all eukaryotic branches that contain dynactin, except for species of the Schizosaccharomycetes clade and some yeast species of the Saccharomyces clade. In the yeast species Vanderwaltozyma polyspora, none of the dynactin subunits were identified, except for dynactin5 and the capping proteins. Vanderwaltozyma 
also does not encode a cytoplasmic dynein homolog. Thus, the $V p$ Dynactin 5 might either be an artefact (unlikely) or it gained a species-specific function outside the dynactin complex (needs experimental verification). The absence of dynein and dynactin in Vanderwaltozyma is most likely related to the specific phenotypic feature that spores are formed by extra mitotic replications after meiosis independent of bud formation [75]. Sole dynactin5 subunits have also been found in Euglenozoa, and dynactin6 additionally in Trypanosoma cruzi. Euglenozoa also contain cytoplasmic dynein heavy and intermediate chains. The presence of only dyanctin 5 (and also dynactin6) is in accordance with the report of a freely soluble pool of these subuntis in cells [19]. In addition, a dynactin5 homolog was found for the plant Vitis vinifera in the cDNA database. This sequence could not be identified in the genome assembly and grouped to a cluster containing parasitic Nematodes in the phylogenetic tree (data not shown) indicating that it is most likely a contamination of the Vitis vinifera cDNA library. Some mammals contain one or more pseudogenes resulting from retro-transcripts.
Dynactin5 from Homo sapiens is encoded in six exons on chromosome 16 (Figure 3D). The available EST and cDNA data do not provide evidence for any alternatively spliced exons in human dynactin5 as well as dynactin5's from any species.

It has been reported that the subunits dynactin4, dynactin5, and Arp11 from mouse, Drosophila, and C. elegans have conserved alkaline pIs [21]. It has been suggested that one or all may interact electrostatically with negatively charged membrane lipids or other acidic cargoes such as lipid droplets or viral nucleocapsids [21]. Recently, dynactin5 from Neurospora crassa was shown to be required for early endosome interaction [76]. However, Drosophila Arp11 and dynactin4 and Arp11 from C. elegans actually have acidic pIs (5.16, 6.61, and 6.4, respectively). These contradictions were perplexing and we decided to determine whether potential electrostatic interactions between dynactin subunits and membranes are conserved across the eukaryotes. Therefore, we have analysed the distribution of $\mathrm{pI}$ values of the pointed-end complex subunits of all species (Figure 4). Dynactin4, dynactin6, and Arp10/Arp11

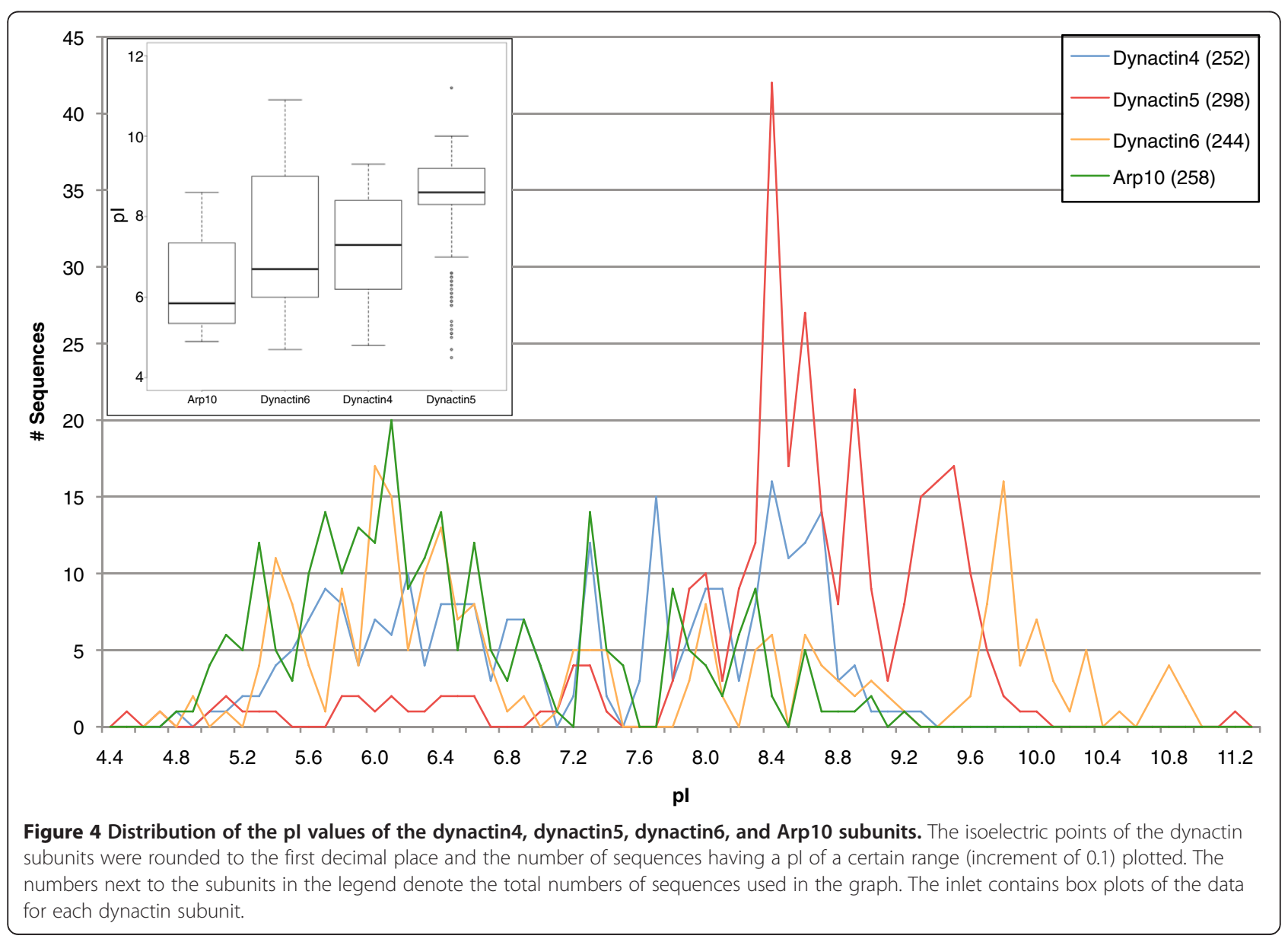


show broad distributions from acidic to alkaline pIs. In contrast, almost all dynactin5 subunits have alkaline pIs suggesting a dynactin5 specific interaction within the complex or to other cellular components. The other pointed-end subunits might have conserved functions that are, however, most likely independent from electrostatic interactions.

\section{Dynactin6}

Dynactin6 is encoded in all eukaryotic branches, except for Aconoidasida (including Plasmodium species), plants, Rhizaria, Bacillariophyta, Saccharomycotina and Schizosaccharomycetes. In the Euteleostei branch (containing part of the fish), the dynactin6 gene has been duplicated. The dynactin6 gene from Homo sapiens is located on chromosome 8 (Figure 3E). It consists of 7 exons all of which are constitutively spliced. Alternatively spliced isoforms have not been identified in any species.

\section{Arp1}

The only species encoding dynactin subunits except Arp1 were the yeast Vanderwaltozyma polyspora DSM 70294, the stramenopiles Aureococcus anophageferens, and the cryptophyte Guillardia theta. Duplicates have been identified in mammals and anole lizard (Additional file 6) grouping to two types, variant A (also known as $\alpha$-centractin, $[77,78]$ ) and variant $\mathrm{B}$ (also known as $\beta$-centractin, [79]). Because the fish Arp1s are most closely related to variant B while the bird and frog Arp1s are most closely related to variant A, the Arp1 duplication event must have been at the origin of the vertebrates, most probably as part of the two whole genome duplications (WGDs) that happened at the emergence of the vertebrates [80]. Unfortunately, EST or genomic DNA data is not available for any Arp1 in Petromyzon marinus. Therefore, we cannot conclude yet whether the Arp1 gene duplication happened at the basis of the vertebrates or the Gnathostomata. Subsequent to the duplication, the ancestors of the fish, birds, and frogs each lost one additional Arp1 paralog, while the mammals and the anole lizard retained both of them. Arp1A and Arp1B have both been shown to be part of dynactin and were found in a constant ratio of about 15:1 in the cytosolic fraction [79]. There was no evidence for a free pool of either isoform and it could not be resolved whether Arp1A and Arp1B appear in distinct or mixed complexes. A recent proteomics study of microtubule associated genes in brain tissue also showed that both paralogs are part of the dynactin pool [81]. Formally it could be possible that all combinations of the two Arp1 paralogs are present in dynactin complexes in mammalian cells. However, because the two paralogs are $90 \%$ identical (96\% similar) and the few differences are distributed over the length of the Arp1 molecule and because most vertebrates retained only one Arp1 homolog it is likely that even mixed dynactin complexes are functionally identical.

\section{Arp10 (Arp11)}

Ten actin-related proteins have been found in the completed genome sequence of Saccharomyces cerevisiae, namely Arp1 to Arp10 [82]. Subsequently, next to Arp1 a second actin-related protein has been identified in the vertebrate dynactin complex. It has been named Arp11 although its closest grouping homologs in a phylogenetic tree of actin and actin-related proteins were the yeast Arp10 and ropy-7 from Neurospora crassa [21]. Most probably, the support for a potential subfamily grouping was not as significant as for other groups of actinrelated proteins. Along the same lines a comparative analysis of 20 completely sequenced eukaryotic genomes did not reveal compelling evidence for grouping Arp10 and Arp11 into one subfamily but recognized that, until then, the appearance of Arp10 and Arp11 was mutually exclusive [83]. It has been suggested that both should be grouped together if yeast Arp10 was found in the dynactin complex or to separate them if both Arp10 and Arp11 were found in a single organism [83]. Recently, yeast Arp10 has been shown to be an integral part of the dynactin complex [41].

Arp10 and Arp11 proteins are very divergent, not only in comparison to the other actin-related proteins but also in between the subfamily. In order to determine their presence or absence in species not encoding unambiguous orthologs we assembled all actin related genes of these species for comparison with complete Arp repertoires of representative organisms. Altogether more than 2,300 Arp proteins have been assembled and analysed including all previously designated Arp classes [83]. Thus, Arp11 orthologs have been identified in the Metazoa, the Fungi (except yeasts), the Amoebozoa, and Oomycetes branch. Arp10 orthologs have been identified in almost all species of the Saccharomycotina branch. Exceptions are Zygosaccharomyces rouxii, Vanderwaltozyma polyspora, Candida glabrata, and Lodderomyces elongisporus. Both Arp 10 and Arp11 have been found in a mutually exclusive manner and group together in the phylogenetic tree of all Arp proteins (Additional file 1). Therefore, and because representatives of both have been shown to be present in dynactin, both are orthologs. According to HUGO this group should be named Arp10 (symbol ACTR10) and Arp11 can be used as synonym [20]. As with the other dynactin genes we will follow the HUGO recommendation and use the name Arp10 for orthologs of this group of actin-related proteins throughout the rest of the analysis. Arp10 has been 


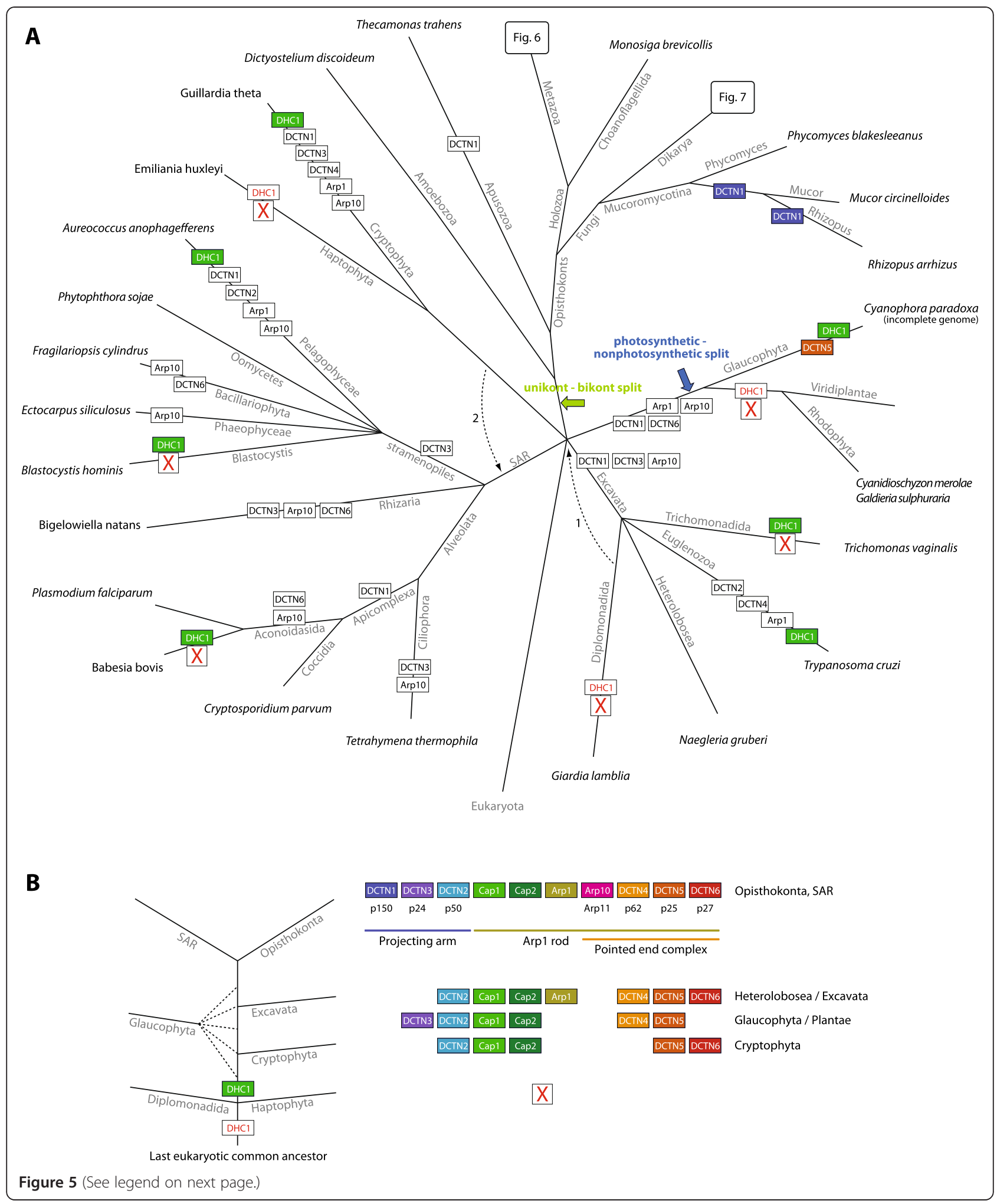


(See figure on previous page.)

Figure 5 Evolution of the dynactin complex with respect to the species evolution. A). The tree represents the most widely accepted phylogenetic tree of the eukaryotes. However, especially the grouping of taxa that emerged close to the origin of the eukaryotes remains highly debated. Therefore, alternative branchings are also indicated in the tree. The phylogeny of the supposed supergroup Excavata is the least understood because only a few species of this branch have been completely sequenced so far. While the grouping of the Heterolobosea, Trichomonada, and Euglenozoa into the Excavata is found in most analyses, the grouping of the Diplomonadida as separate phylum or as part of the Excavata is still debated (arrow 1 [84]). According to most of the recent phylogenetic analyses, the Alveolata, Rhizaria, and Stramenopiles form the superfamily SAR $[85,86]$. The placement of the Haptophyceae and Cryptophyta to the SAR is still highly debated. Although several analyses are in favour of this grouping (arrow 2; [87-89]) most analyses are in contrast [85,86,90-92]. At each leaf of the tree one representative species of the branch is printed. Branch lengths are arbitrary. The tree illustrates the presence and absence of each subunit of the dynactin complex in the corresponding species under the hypothesis of five eukaryotic supergroups and the position of the LECA as indicated. Alternative eukaryotic roots are indicated by coloured arrows. Coloured boxes show gene duplications and white boxes show gene loss events of dynactin subunits. The presence (green box) or absence (white box) of the cytoplasmic dynein heavy chain gene DHC1 is indicated for those species that do not have dynactin or miss most of the subunits. B) A possible tree of some major branches of the eukaryotes is shown together with the subunits encoded by the respective taxa. The tree is based on the most parsimonious way the branches could have diverged based on the assumption that during this evolution subunits have only been gained and not lost.

duplicated in Gallus gallus, and two Arp10 homologs were identified in the pseudotetraploid Xenopus laevis. In the other branches of the eukaryotes, none of the assembled actin-related proteins clearly belongs to the Arp10 subfamily.

\section{Capping proteins Capa (Cap1) and Cap $\beta$ (Cap2)}

The ubiquitous actin capping proteins Cap $\alpha$ (Cap1) and Cap $\beta$ (Cap2) are part of the dynactin complex but except for capping the Arp1 minifilament they do not seem to have dynactin-specific functions and will therefore be discussed in Additional file 7.

\section{Discussion}

Here, we have performed an exhaustive analysis of all known dynactin subunits in all eukaryotic genomes available until September 2011. The presence of dynactin subunits is always coupled to the presence of a cytoplasmic dynein heavy chain (DHC1). Some branches do not contain a DHC1 and accordingly do not contain any dynactin subunit (Figure 5A): plants, diplomonads (e.g. Giardia lamblia), Haptophyceae (e.g. Emiliania huxleyi), Entamoebidae, some of the Microsporidia, and Rhodophyta (e. g. Cyanidioschyzon merolae and Galdieria sulphuraria). While the presence of dynactin is coupled to the presence of a DHC1 there are a few species that contain cytoplasmic dyneins but do not encode dynactin subunits: Piroplasmida (e.g. Babesia and Theileria species), some Microsporidia, and Parabasalia (e.g. Trichomonas vaginalis). The DHC1s of the known Piroplasmida and Microsporidia are, however, extremely divergent and shortened (about 3,200 instead of the usual 4,500 residues) and it is not known whether these are functional motors at all. Together, these results demonstrate the strong functional interconnection between dynactin and cytoplasmic dynein. In addition, both were most probably already present in the last common ancestor of the eukaryotes. Although dynein-independent functions have been reported for dynactin these are most likely subfunctionalisations in specific branches of the eukaryotic tree in which either dyneins partnership became obsolete for certain functions or in which dynactin acquired additional specific binding partners.

All of dynactins known eleven subunits were already present in the last common ancestor of the eukaryotes because all of them have been identified in at least two of the major lineages (Figure 5A). However, in many genomes single subunits are missing. Is this due to gene loss events or due to problems in their identification? The dynactin complex and the dynactin subunits have first been identified and characterised in vertebrates and insects, and these constitute the reference sequences. It could be possible, that some subunits have not been identified in several branches and species, which diverged very early in eukaryotic evolution, because of their low similarity to the subunits of the metazoan species that prevented their identification. However, unambiguous homologs have been identified and annotated in every major lineage of the eukaryotes demonstrating that the sequence similarity in general dates back to the last common ancestor. Even when we searched with these homologs instead of the reference sequences, missing homologs in closely related species could not be identified. For example, although a dynactin1 has been found in Tetrahymena we were not able to identify dynactin1 homologs in Toxoplasma gondii, Plasmodium and Cryptosporidium species. Therefore, we rather assume that subunits have been lost during evolution although we cannot exclude that we might have missed divergent homologs that can only be revealed in experiments, but not sequence based analyses. In addition, subunits might be missing because of gaps in the sequence assemblies.

\section{Evolution of the dynactin complex in eukaryotes}

The evolution of the dynactin complex in eukaryotes is characterised by many branch- and species-specific gene 
loss and gene duplication events (Figure 5A). The monophyly of the SAR branch is well established now [93] as well as the monophyly of the Opisthokonts (and even unikonts, [94]) and Excavata [94]. The last common ancestors of both the SAR and the unikonts contained all eleven dynactin subunits (Figure 5B). If the unikont-bikont hypothesis, that combines all major kingdoms except the unikonts into a supergroup called bikonts and places the eukaryotic root between these two supergroups [93,95], were true the last common ancestor of all extant eukaryotes (LECA) must have contained the complete dynactin complex (Figure 5A). Another hypothesis places the origin of the eukaryotes between Plantae and the rest (photosynthetic-nonphotosynthetic split, [96,97]). Unfortunately, the genome sequence of the Glaucophyte Cyanophora paradoxa is not complete, but it seems that based on the latter hypothesis the LECA would have contained only dynactin2, dynactin3, dynactin4, dynactin5, and the CAP proteins. The dynactin data does not help in resolving the issue of unambiguously placing the eukaryotic root because its analysis involves eleven subunits and is biased by the very small number of sequenced species in the taxa Cryptophyta, Haptophyta, Glaucophyta and Excavata except Euglenozoa. Thus it could be possible that more complete dynactin inventories will be found in newly sequenced species of these taxa like in the SAR branch in which all dynactin subunits were found in total but not in a single species. Building a parsimonious tree from the presence and absence of the dynactin subunits alone in all species is not possible without breaking established monophyletic groups like the sistergroups Fungi and Holozoa, or the sistergroups Blastocystis and Oomycetes. However, if we try to reconstruct a tree of the eukaryotes based on the major taxa by only breaking the still debated phylogenetic groupings of the Haptophyta and the Diplomonadida but leaving established supergroups intact, the following scenario can be imagined (Figure 5B). Diplomonadida and Haptophyta both do not contain cytoplasmic dynein and dynactin and were therefore the first to diverge in eukaryotic evolution. The LECA would have not contained dynein and dynactin in this case. Next, the dynactin 5 and dynactin 6 subcomplex and dynactin 2 were invented and the Cryptophyta separated. This would be consistent with the finding of a freely soluble pool of dynactin 5 and dynactin 6 in cells [19]. The placing of the Glaucophyta (as part of the Plantae) is not yet clear due to the incomplete genome of the single representative Cyanophora paradoxa. The Glaucophyta do have already dynactin3 and dynactin4 but miss dynactin6. Subsequently, Arp1 and dynactin4 evolved completing the Arp1 rod in Heterolobosea (Excavata). Finally, the projecting arm had been completed in SAR and Opisthokonta. However, this model is based on the assumption that dynactin subunits had only been gained and not lost during early eukaryotic evolution, and the model contradicts the unikont-bikont and the photosynthetic-nonphotosynthetic split hypotheses. Given the many dynactin gene loss events in later separating branches it is more likely that the LECA already contained all dynactin subunits. This assumption could be combined with both split hypotheses and is in agreement with analyses of other protein complexes in which the reconstructed complexes of the LECA contained most of the present-day subunits [98,99].

From the stage of the SAR and Opisthokonta the subsequent evolution of the branches is determined by many and specific gene loss events. Especially Arp10, dynactin6, dynactin1, and dynactin3 have been lost independently in many branches. The Arp1 filament capping function of Arp10 might have been taken over by one of the so far unclassified actin-related proteins or dynactin4. Dynactin6 forms a tight complex with dynactin5 in vertebrates [21] but because also yeasts have, if at all, only dynactin 5 it might be possible that dynactin 5 forms a homodimer in the species lacking dynactin6. Dynactin1 subunits have independently been lost by many species or their dynactin 1 homologs have lost the CAP-Gly domain hindering their identification because of the low sequence similarity of the coiled-coil regions. Vertebrate and Drosophila dynactin1 transcripts without a CAP-Gly domain (corresponding to "p135") are very well versed to bridge the Arp1 rod to dynein and microtubules showing similar intracellular trafficking of organelles $[15,58,100]$. Thus, so far unknown dynactin1s without CAP-Gly domains could still be present in Apicomplexa, Heterolobosea and Apusozoa. Dynactin3 is necessary for the incorporation of dynactin1 into the yeast dynactin complex [40]. However, it has been shown that dynactin1 in vertebrates and Drosophila contains an independent Arp1 binding site, and therefore dynactin3 might not be essential for the dynactin complex in all species. This might explain dynactin3's absence in many branches that have dynactin1. Other reasons could be that we were not able to identify all dynactin 3 subunits because of their low sequence conservation or that dynactin3 has diverged in independent branches so far that homology cannot be detected any more. In any case, strong changes happened to this subunit independently in many early branching eukaryotic lineages and also in closely related branches.

\section{Expansion of dynactin complexity in metazoa}

Dynactin complex diversity in metazoa is greatly enhanced by branch specific gene duplications and the introduction of alternative splice forms (Figure 6). The dynactin 1 gene has been duplicated independently in the nematods of the Spirurida branch, in the Brachycera including the Drosophila species [48], and in fish genomes (Figure 6). Thus, dynactin complexes with different properties could be generated in these species by 


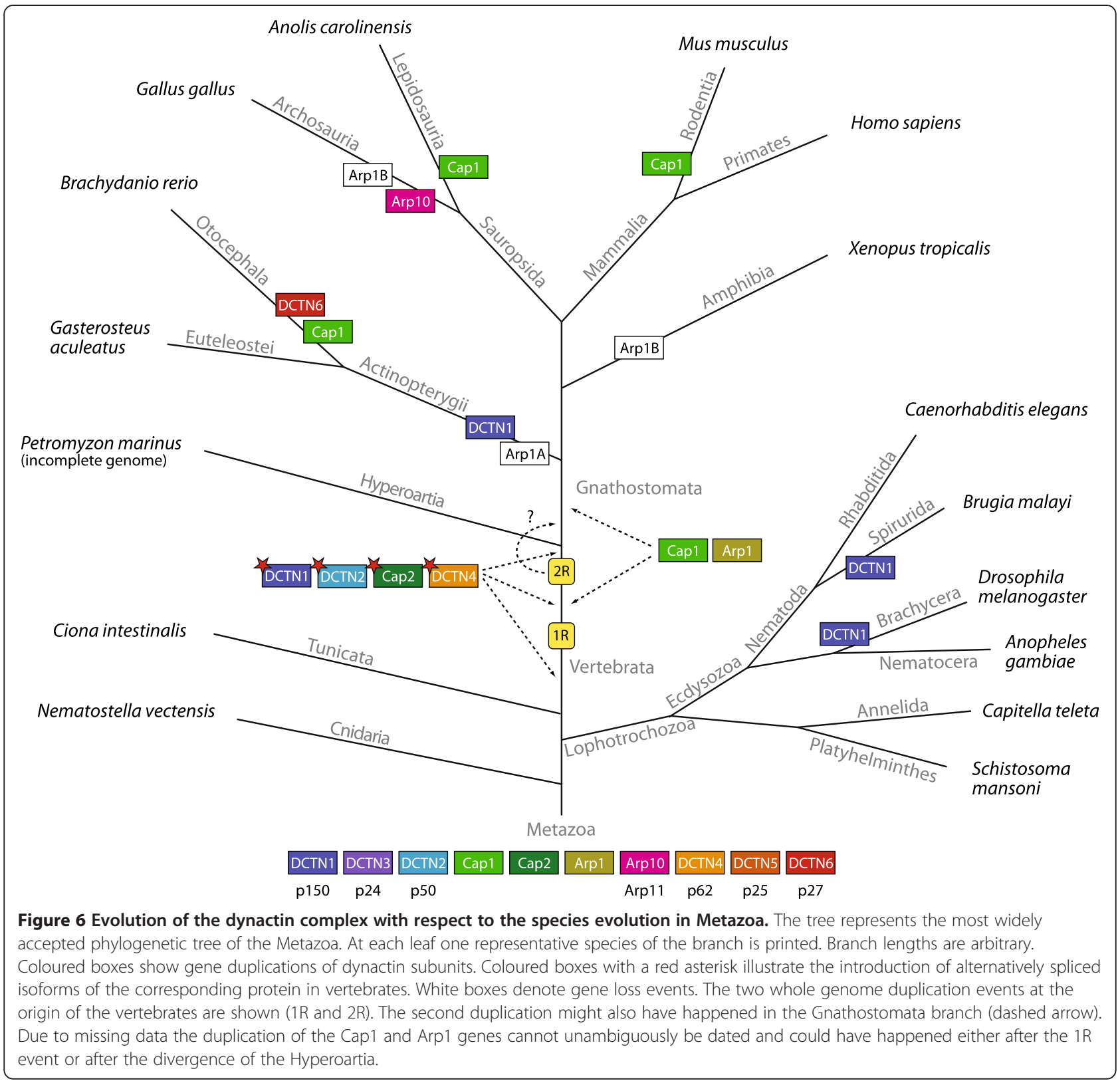

assembling two different homodimers or a heterodimer of their different dynactin1 subunits. Dynactin6 and Arp10 have also been duplicated in the Otocephala branch (including Brachydanio rerio) and in birds, respectively. Arp1 has been duplicated early in vertebrate history and subsequently fish, birds, and amphibians lost different types of the duplicates. An alternative but less likely scenario would be that all dynactin subunit duplications were part of the two whole genome duplications at the origin of the vertebrates followed by numerous independent gene losses in the extant species.

Interestingly, alternatively spliced exons have been invented in vertebrate dynactin1, dynactin2, and dynactin 4 genes either before, in between or after the two whole genome duplication events happened but before the divergence of the agnaths and the Gnathostomata (Figure 6). Thus, complexity and fine-tuning of dynactin1 can considerably be enhanced by differential inclusion of four exons that can be combined with three alternative start sites for translation (Figure 2). The alternative start sites affect the inclusion/exclusion of the CAP-Gly domain, and three of the alternative exons encode consecutive pieces of the basic region between the CAP-Gly domain and the first coiled-coil domain. These alternative splice forms therefore do not affect the binding of dynactin to cytoplasmic dynein but only the region attaching dynactin to microtubules [101]. Altogether, 36 different transcripts can theoretically be generated for each vertebrate 
dynactin1 gene. It is not known yet whether heterodimers of dynactin1 isoforms are possible, which would multiply the theoretically possible number of different dynactin complexes. However, it seems unlikely that a transcript with certain functionality, e.g. a transcript without the CAP-Gly domain, would be combined with a subunit that could in part reconstitute the missing function. This conclusion is consistent with findings in rat brain that showed distinct complexes of dynactin with either full-length dynactin1 (p150) or CAP-Gly diminished dynactin1 (p135, [100]). The three alternative splice forms analysed so far (p150- $\Delta 5 ; \mathrm{p} 150-\Delta 5,6: \mathrm{p} 150-\Delta 5,6,7)$ also showed a tissue specific expression pattern [58] demonstrating that most likely only a limited number of different dynactin1 subunits and not all possible combinations are present in a single cell. However, all combinations will most likely be present in each organism.

Because most subunits are present in multiple copies in the complex, a single gene duplication of one subunit would already result in two, three, or more different complexes, if complexes were built not only from distinct but also mixed subunit compositions. Based on their gene content, the vertebrates can theoretically build thousands of different dynactin complexes considering all combinations of the genes and splice forms. However, most of the differences are introduced by tiny changes. For example, all identified alternatively spliced exons contain only between two and seven residues. In addition, the two Arp1 paralogs differ in only a few residues that are distributed over the length of the molecule. Thus, these small changes are not expected to considerably alter the overall structure of dynactin. However, it is well known that even single posttranslational modifications can dramatically change the functions of proteins from activating/deactivating enzymes or binding/non-binding other proteins or membranes (e.g. phosphorylation of dynactin1 strongly reduces its microtubule affinity, [102]). Concerning the two Arp1 paralogs it is hard to imagine how defined combinations could be generated in the cell given that eight to nine Arp1 subunits comprise the Arp1 minifilament. This would require a strong regulation of the protein level of both paralogs as well as a strong regulation of the position-specific incorporation into the minifilament. The 15:1 ratio of the paralogs could be regulated at the transcription level but it is very likely that both are just randomly incorporated into dynactin without influencing its structure, stability, and function. Therefore, dynactins functions will most likely only be modulated through the various alternative transcripts. The differences seem small but have not been studied at a molecular level yet.

\section{Reduction of dynactin complexity in yeasts}

In general, gene loss in yeasts only affects the pointedend complex subunits and dynactin3, which mediates association of dynactin1 to dynactin2 (Figure 7) The Schizosaccharomycetes and Saccharomycotina both have lost the dynactin6 subunit. Dynactin 5 and dynactin6 are predicted to fold into left-handed $\beta$-helical structures [103], and are supposed to form a tight heterodimeric complex in vertebrates [21]. They show low sequence similarity but can still be aligned to each other (data not shown). Therefore, it could be possible that dynactin 5 forms homodimers in those species that do not encode dynactin6. Dynactin3 and dynactin5 have been lost in Schizosaccharomycetes and many Saccharomycotina subbranches. The loss of dynactin3 in yeasts is surprising because it has been found to be essential to recruit dynactin 1 to the dynactin complex in Saccharomyces cerevisiae [40]. The dynactin1 genes in yeasts have about the same lengths, which is also true for the dynactin 2 genes. A missing dynactin 3 is therefore not compensated by additional domains in the other dynactin subunits. Either changes at the surface of dynactin 1 or dynactin 2 may supersede dynactin3 or we were not able to detect the missing dynactin3 subunits yet. Dynactin5 is required for the interaction of dynein with a subset but not all membranous vesicles, which is supported by the conserved basic pI of all dynactin 5 subunits and by membraneflotation essays [76]. This is also consistent with findings in Saccharomyces cerevisiae, which does not encode a dynactin 5 subunit, that dynein is necessary for nuclear migration and spindle orientation but does not perform vesicle transport [104,105]. In addition, dynactin4, Arp10, and dynactin2 have been lost in several, two, and 1 branch of the yeasts, respectively. Arp10 is needed for the stability and capping of the Arp1 filament [41] and its absence should thus affect dynactins integrity. Both Zygosaccharomyces and Lodderomyces lack Arp10 and dynactin4, the other pointed-end capping protein and it is unclear how the Arp1 filament could be stabilized in these species.

\section{Conclusions}

The dynactin complex is a very ancient complex that already existed in the last common ancestor of extant eukaryotes. It consists of eleven subunits of which at least seven comprise the core structure: dynactin1, dynactin2, dynactin4, dynactin5, the heterodimeric capping protein, and Arp1. The presence of the dynactin complex coincides with that of the cytoplasmic dynein heavy chain: Organisms that do not encode cytoplasmic dyneins like plants and diplomonads also do not encode dynactin subunits either. In the metazoan lineage, several of the dynactin subunits were duplicated independently in different branches. The largest repertoire is found in vertebrates. Also at the origin of the vertebrates, several alternatively spliced exons have 


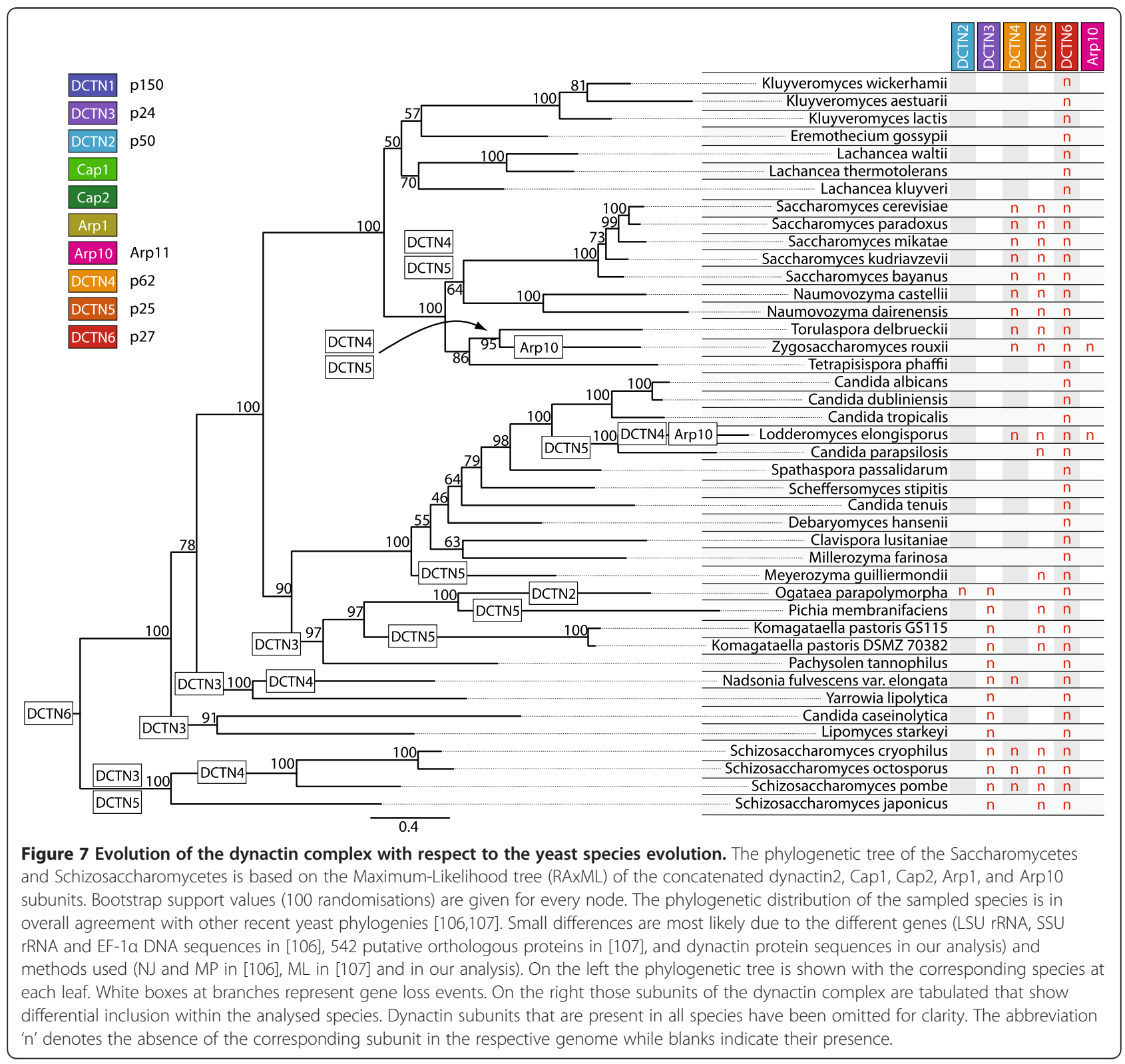

been invented providing the basis for modulating the core functions. The most prominent example is the dynactin1 gene, from which 36 different transcripts could be generated. In contrast, ascomycetous yeasts have reduced subunit compositions. In general they have reduced pointed-end complexes, which in return let to the loss of the functions coupled to the specific subunits.

\section{Methods}

\section{Identification and annotation of the genes of the dynactin subunits}

Dynactin genes have been identified in iterated TBLASTN and PSI-BLAST searches of the completed or almost completed genomes of about 600 organisms starting with the protein sequences of the human dynactin subunits. All hits were manually analysed at the genomic DNA level. The correct coding sequences were identified with the help of multiple sequence alignments of the respective dynactin subunits. As the amount of dynactin sequences increased (especially the number of sequences from taxa with few representatives), many of the initially predicted sequences were reanalysed to correctly identify all exon borders. Where possible, EST data has been analysed to help in the annotation process. In addition to the analysis of these large-scale sequencing projects, all dynactin sequences in the "nr" database at NCBI have been collected and reanalysed.

Several of the genes contain alternative splice forms. The different splice forms were not considered independently 
in the analysis but in all cases the same splice forms were taken for homologous dynactin proteins. All sequence related data (names, corresponding species, GenBank ID's, alternative names, corresponding publications, domain predictions, sequences, and gene structure reconstructions) and references to genome sequencing centers are available through the CyMoBase (http://www.cymobase. org, [108]). A list of the species analysed, their abbreviations as used in the alignments and trees, as well as detailed information and acknowledgments of the respective sequencing centers is also available as Additional file 8 . Webscipio $[53,109]$ was used to reconstruct the gene structure (exon/intron pattern) of each sequence.

\section{Generating the multiple sequence alignment}

The multiple sequence alignments of the dynactin subunits have been built and extended during the process of annotating and assembling new sequences. The initial alignments have been generated from the first about 20 sequences obtained from NCBI using the ClustalW software with standard settings [110]. During the following correction of the sequences (removing wrongly annotated sequences and filling gaps) the alignment has been adjusted manually. Subsequently, every newly predicted sequence has preliminarily been aligned to its supposed closest relative using ClustalW, the aligned sequence added to the multiple sequence alignment of the respective dynactin subunit, and the alignment adjusted manually during the subsequent sequence validation process. Still, many gaps in sequences derived from low-coverage genomes remained. In those cases, the integrity of the exons next to gaps has been maintained (gaps in the genomic sequence are reflected as gaps in the multiple sequence alignment). The sequence alignments of the dynactin subunits can be obtained from CyMoBase or Additional file 1.

\section{Comparison of the sequence identities and similarities}

Sequences designated "Fragment", "Partial", or "Pseudogene" were removed from the multiple sequence alignments of the dynactin subunits. Poorly aligned positions and divergent regions of the alignments were removed using Gblocks [111] with the following parameters: A) The minimum number of sequences for a conserved position and the minimum of sequences for a flank position were set to the minimum (e.g. half the number of sequences plus one). B) The maximum number of contiguous nonconserved positions was set to 32000 and the minimum length of a block was set to 2 . C) The parameter for the allowed gap position was set to 'all'.

Sequence identity matrices (2D-matrix tables containing sequence identities scores for each pair of sequences) were calculated for each alignment using the method implemented in BioEdit (Tom Hall, http://www.mbio. ncsu.edu/bioedit/bioedit.html). Shortly, the reported numbers represent the ratio of identities to the length of the longer of the two sequences after positions where both sequences contain a gap are removed. Sequence similarity matrices were calculated with MatGAT [112] using the BLOSUM62 substitution matrix and setting the gap opening and extending penalties to 12 and 2, respectively.

\section{Computing and visualising phylogenetic trees}

For calculating phylogenetic trees of single dynactin subunits only complete and partial sequences were included in the dataset. For the calculation of the tree of the yeast species, the sequences of the Arp1, Arp10, Cap1, Cap2, and dynactin2 subunit of each species of the Saccharomyces and the Schizosaccharomyces branch were concatenated. Missing protein sequences (Zygosaccharomyces rouxii Arp10, Lodderomyces elongisporus Arp10, Ogataea parapolymorpha dynactin2, and Naumovozyma dairenensis Cap2) were substituted by gaps. The phylogenetic trees were generated using two different methods for each dataset: 1 . ProtTest was used to determine the most appropriate of the available 112 possible amino acid substitution models [113]. The tree topology was calculated with the BioNJ algorithm and both the branch lengths and the model of protein evolution were optimized simultaneously. The Akaike Information Criterion with a modification to control for small sample size (AICc, with alignment length representing sample size) identified the LG model [114] to be the best for the dynactin3, dynactin5, dynactin6, Arp, and Cap datasets and the JTT model [115] for dynactin1, dynactin2, and dynactin4. Maximum likelihood (ML) analysis with estimated proportion of invariable sites and bootstrapping (1,000 replicates) were performed using RAxML [116]. 2. Posterior probabilities were generated using MrBayes v3.1.2 [117] with the MPI option [118]. Two independent runs with 5,000,000 generations, four chains, and a random starting tree were computed using the mixed amino-acid option. MrBayes used the WAG model [119] for all protein alignments. Trees were sampled every 1.000th generation and the first $25 \%$ of the trees were discarded as "burn-in" before generating a consensus tree. Phylogenetic trees were visualized with the CLC Sequence Viewer (http://www. clcbio.com) and FigTree (http://tree.bio.ed.ac.uk/software/ figtree/) and are available as Additional file 1.

\section{Additional files}

Additional file 1: Zip archive of the Maximum Likelihood and Bayesian inference trees, and the sequence alignments of the dynactin subunits. The file includes all Maximum Likelihood and Bayesian trees of all dynactin proteins in the Newick format. The sequence alignments of the proteins are included in fasta format.

Additional file 2: Dynactin inventory of the analysed species. The file lists the presence and number of orthologs for each dynactin subunit 
for each analysed organism in taxonomic order.

Additional file 3: Phylogenetic tree of dynactin1. The file contains the phylogenetic tree of dynactin 1 highlighting the species- and branchspecific gene duplication events.

Additional file 4: Detailed description of the pseudo-transcripts of dynactin4. The file contains details about the pseudo-transcripts of dynactin4.

Additional file 5: Sequence alignment of fungal dynactin4 proteins. The file contains the sequence alignment of the $\mathrm{N}$-termini of several fungal dynactin4 (p62) subunits showing that the upstream methionines in Neurospora and Sordaria are most likely not the translation start sites.

Additional file 6: Phylogenetic tree of Arp1. The file contains the phylogenetic tree of Arp 1 focused on the vertebrate branch highlighting the Arp1 gene duplication event and subsequent branch-specific losses of Arp1 subtypes.

\section{Additional file 7: Discussion of the evolution of the Cap proteins.}

The file contains a discussion of the gene duplications, which happened to the Capa (Cap1) subunit, and of alternative splice variants of the Cap $\beta$ (Cap2) subunit [120,121].

Additional file 8: Species table. The file contains all species of the analysis, their scientific names, the abbreviation as used in the sequence alignments and trees, the species taxonomy, references to sequencing centers, and publications if genome analyses have already been published.

\section{Competing interests}

The authors declare that they have no competing interests.

\section{Authors' contributions}

$\mathrm{BH}$ performed all database related work, adjusted the CyMoBase software for specific dynactin related needs, did all data analysis and drafted the manuscript. MK assembled and annotated all sequences, and assisted in writing the manuscript. Both authors read and approved the final version of the manuscript.

\section{Acknowledgements}

First we would like to thank the Editor Henner Brinkmann and the anonymous reviewers for their very constructive comments that helped to considerably improve the manuscript. We also would like to thank the many sequencing centers and funding agencies for making unpublished sequence data available. Last, we want to thank Dr. Florian Odronitz and Klas Hatje for their help with CyMoBase and WebScipio, and Prof. Christian Griesinger for his continuous generous support. Sebastian Becker helped in the annotation of some of the sequence. This work has been funded by grants KO 2251/3-1 and KO 2251/3-2 of the Deutsche Forschungsgemeinschaft

Received: 27 January 2012 Accepted: 22 June 2012

Published: 22 June 2012

\section{References}

1. Gill SR, Schroer TA, Szilak I, Steuer ER, Sheetz MP, Cleveland DW: Dynactin, a conserved, ubiquitously expressed component of an activator of vesicle motility mediated by cytoplasmic dynein. J Cell Biol 1991, 115:1639-1650.

2. Schroer TA, Sheetz MP: Two activators of microtubule-based vesicle transport. J Cell Biol 1991, 115:1309-1318.

3. Steffen W, Karki S, Vaughan KT, Vallee RB, Holzbaur EL, Weiss DG, Kuznetsov SA: The involvement of the intermediate chain of cytoplasmic dynein in binding the motor complex to membranous organelles of Xenopus oocytes. Mol Biol Cell 1997, 8:2077-2088.

4. Holleran EA, Ligon LA, Tokito M, Stankewich MC, Morrow JS, Holzbaur ELF: BIII Spectrin Binds to the Arp1 Subunit of Dynactin. J Biol Chem 2001, 276:36598-36605.

5. King SJ, Schroer TA: Dynactin increases the processivity of the cytoplasmic dynein motor. Nat Cell Biol 2000, 2:20-24.

6. Ross JL, Wallace K, Shuman H, Goldman YE, Holzbaur EL: Processive bidirectional motion of dynein-dynactin complexes in vitro. Nat Cell Biol 2006, 8:562-570.
7. Culver-Hanlon TL, Lex SA, Stephens AD, Quintyne NJ, King SJ: A microtubule-binding domain in dynactin increases dynein processivity by skating along microtubules. Nat Cell Biol 2006, 8:264-270.

8. Kardon JR, Reck-Peterson SL, Vale RD: Regulation of the processivity and intracellular localization of Saccharomyces cerevisiae dynein by dynactin Proc Natl Acad Sci USA 2009, 106:5669-5674

9. Quintyne NJ, Schroer TA: Distinct cell cycle-dependent roles for dynactin and dynein at centrosomes. J Cell Biol 2002, 159:245-254.

10. Quintyne NJ, Gill SR, Eckley DM, Crego CL, Compton DA, Schroer TA Dynactin Is Required for Microtubule Anchoring at Centrosomes. J Cell Biol 1999, 147:321-334.

11. Eaton BA, Fetter RD, Davis GW: Dynactin is necessary for synapse stabilization. Neuron 2002, 34:729-741.

12. Howell BJ, McEwen BF, Canman JC, Hoffman DB, Farrar EM, Rieder CL, Salmon ED: Cytoplasmic dynein/dynactin drives kinetochore protein transport to the spindle poles and has a role in mitotic spindle checkpoint inactivation. J Cell Biol 2001, 155:1159-1172.

13. Lee $\mathrm{WL}$, Oberle JR, Cooper JA: The role of the lissencephaly protein Pac1 during nuclear migration in budding yeast. J Cell Biol 2003, 160:355-364

14. Merdes A, Ramyar K, Vechio JD, Cleveland DW: A Complex of NuMA and Cytoplasmic Dynein Is Essential for Mitotic Spindle Assembly. Cell 1996, 87:447-458.

15. Kim H, Ling S-C, Rogers GC, Kural C, Selvin PR, Rogers SL, Gelfand VI: Microtubule binding by dynactin is required for microtubule organization but not cargo transport. J Cell Biol 2007, 176:641-651.

16. Deacon SW, Serpinskaya AS, Vaughan PS, Fanarraga ML, Vernos I, Vaughan $K T$, Gelfand VI: Dynactin is required for bidirectional organelle transport. J Cell Biol 2003, 160:297-301.

17. Berezuk MA, Schroer TA: Dynactin enhances the processivity of kinesin-2. Traffic 2007, 8:124-129.

18. Blangy A, Arnaud L, Nigg EA: Phosphorylation by p34cdc2 protein kinase regulates binding of the kinesin-related motor $\mathrm{HsEg} 5$ to the dynactin subunit p150. J Biol Chem 1997, 272:19418-19424.

19. Schroer TA: Dynactin. Annu Rev Cell Dev Biol 2004, 20:759-779.

20. Seal RL, Gordon SM, Lush MJ, Wright MW, Bruford EA: Genenames.org: the HGNC resources in 2011. Nucleic Acids Res 2011, 39:D514-D519.

21. Eckley DM, Gill SR, Melkonian KA, Bingham JB, Goodson HV, Heuser JE, Schroer TA: Analysis of dynactin subcomplexes reveals a novel actinrelated protein associated with the arp1 minifilament pointed end. J Cell Biol 1999, 147:307-320.

22. Schafer DA, Gill SR, Cooper JA, Heuser JE, Schroer TA: Ultrastructural analysis of the dynactin complex: an actin-related protein is a component of a filament that resembles F-actin. J Cell Biol 1994, 126:403-412.

23. Waterman-Storer CM, Karki S, Holzbaur EL: The p150Glued component of the dynactin complex binds to both microtubules and the actin-related protein centractin (Arp-1). Proc Natl Acad Sci U S A 1995, 92:1634-1638.

24. Akhmanova A, Hoogenraad CC: Microtubule plus-end-tracking proteins: mechanisms and functions. Curr Opin Cell Biol 2005, 17:47-54.

25. Weisbrich A, Honnappa S, Jaussi R, Okhrimenko O, Frey D, Jelesarov I, Akhmanova A, Steinmetz MO: Structure-function relationship of CAP-Gly domains. Nat Struct Mol Biol 2007, 14:959-967.

26. Vaughan KT, Vallee RB: Cytoplasmic dynein binds dynactin through a direct interaction between the intermediate chains and p150Glued. J Cell Biol 1995, 131:1507-1516.

27. Karki S, Holzbaur EL: Affinity chromatography demonstrates a direct binding between cytoplasmic dynein and the dynactin complex. J Biol Chem 1995, 270:28806-28811.

28. Echeverri CJ, Paschal BM, Vaughan KT, Vallee RB: Molecular characterization of the 50-kD subunit of dynactin reveals function for the complex in chromosome alignment and spindle organization during mitosis. J Cell Biol 1996, 132:617-633.

29. Jacquot $G$, Maidou-Peindara $P$, Benichou S: Molecular and functional basis for the scaffolding role of the p50/dynamitin subunit of the microtubule-associated dynactin complex. J Biol Chem 2010, 285:23019-23031.

30. Melkonian KA, Maier KC, Godfrey JE, Rodgers M, Schroer TA: Mechanism of Dynamitin-mediated Disruption of Dynactin. J Biol Chem 2007, 282:19355-19364.

31. Terasawa M, Toya M, Motegi F, Mana M, Nakamura K, Sugimoto A: Caenorhabditis elegans ortholog of the p24/p22 subunit, DNC-3, is 
essential for the formation of the dynactin complex by bridging DNC-1 /p150Glued and DNC-2/dynamitin. Genes Cells 2010, 15:1145-1157.

32. Holleran EA, Tokito MK, Karki S, Holzbaur EL: Centractin (ARP1) associates with spectrin revealing a potential mechanism to link dynactin to intracellular organelles. J Cell Biol 1996, 135:1815-1829.

33. Caviston JP, Holzbaur ELF: Microtubule motors at the intersection of trafficking and transport. Trends Cell Biol 2006, 16:530-537.

34. Lee $\mathrm{H}$, Kumar S, Plamann M: Null Mutants of the Neurospora Actinrelated Protein 1 Pointed-End Complex Show Distinct Phenotypes. Mol Biol Cell 2001, 12:2195-2206.

35. Plamann M, Minke PF, Tinsley JH, Bruno KS: Cytoplasmic dynein and actinrelated protein Arp1 are required for normal nuclear distribution in filamentous fungi. J Cell Biol 1994, 127:139-149.

36. Robb MJ, Wilson MA, Vierula PJ: A fungal actin-related protein involved in nuclear migration. Mol Gen Genet 1995, 247:583-590.

37. Vierula PJ, Mais JM: A gene required for nuclear migration in Neurospora crassa codes for a protein with cysteine-rich, LIM/RING-like domains. Mol Microbiol 1997, 24:331-340.

38. Tinsley JH, Minke PF, Bruno KS, Plamann M: p150Glued, the largest subunit of the dynactin complex, is nonessential in Neurospora but required for nuclear distribution. Mol Biol Cell 1996, 7:731-742.

39. Bruno KS, Tinsley JH, Minke PF, Plamann M: Genetic interactions among cytoplasmic dynein, dynactin, and nuclear distribution mutants of Neurospora crassa. Proc Natl Acad Sci U S A 1996, 93:47754780.

40. Amaro IA, Costanzo M, Boone C, Huffaker TC: The Saccharomyces cerevisiae Homolog of p24 Is Essential for Maintaining the Association of p150Glued With the Dynactin Complex. Genetics 2008, 178:703-709.

41. Clark SW, Rose MD: Arp10p is a pointed-end-associated component of yeast dynactin. Mol Biol Cell 2006, 17:738-748.

42. Kahana JA, Schlenstedt G, Evanchuk DM, Geiser JR, Hoyt MA, Silver PA The yeast dynactin complex is involved in partitioning the mitotic spindle between mother and daughter cells during anaphase $B$. Mol Biol Cell 1998, 9:1741-1756.

43. Muhua L, Karpova TS, Cooper JA: A yeast actin-related protein homologous to that in vertebrate dynactin complex is important for spindle orientation and nuclear migration. Cell 1994, 78:669-679.

44. Holzbaur ELF, Hammarback JA, Paschal BM, Kravit NG, Pfister KK, Vallee RB: Homology of a $150 \mathrm{~K}$ cytoplasmic dynein-associated polypeptide with the Drosophila gene Glued. Nature 1991, 351:579-583.

45. Eckley DM, Schroer TA: Interactions between the Evolutionarily Conserved, Actin-related Protein, Arp11, Actin, and Arp1. Mol Biol Cell 2003, 14:2645-2654.

46. Skop AR, White JG: The dynactin complex is required for cleavage plane specification in early Caenorhabditis elegans embryos. Curr Biol 1998, 8:1110-1116.

47. Gönczy P, Pichler S, Kirkham M, Hyman AA: Cytoplasmic dynein is required for distinct aspects of $M T O C$ positioning, including centrosome separation, in the one cell stage Caenorhabditis elegans embryo. J Cell Biol 1999, 147:135-150.

48. Odronitz F, Becker S, Kollmar M: Reconstructing the phylogeny of 21 completely sequenced arthropod species based on their motor proteins. BMC Genomics 2009, 10:173.

49. Yandell M, Ence D: A beginner's guide to eukaryotic genome annotation Nat Rev Genet 2012, 13:329-342.

50. Odronitz F, Kollmar M: Drawing the tree of eukaryotic life based on the analysis of 2,269 manually annotated myosins from 328 species. Genome Biol 2007, 8:R196.

51. Eckert C, Hammesfahr B, Kollmar M: A holistic phylogeny of the coronin gene family reveals an ancient origin of the tandem-coronin, defines a new subfamily, and predicts protein function. BMC Evol Biol 2011, 11:268

52. Kollmar M, Lbik D, Enge S: Evolution of the eukaryotic ARP2/3 activators of the WASP family: WASP, WAVE, WASH, and WHAMM, and the proposed new family members WAWH and WAML. BMC Res Notes 2012, 5:88.

53. Odronitz F, Pillmann H, Keller O, Waack S, Kollmar M: WebScipio: an online tool for the determination of gene structures using protein sequences. BMC Genomics 2008, 9:422.

54. Gupta KK, Joyce MV, Slabbekoorn AR, Zhu ZC, Paulson BA, Boggess B, Goodson HV: Probing interactions between CLIP-170, EB1, and microtubules. J Mol Biol 2010, 395:1049-1062.
55. Tian G, Lewis SA, Feierbach B, Stearns T, Rommelaere H, Ampe C, Cowan NJ: Tubulin subunits exist in an activated conformational state generated and maintained by protein cofactors. J Cell Biol 1997, 138:821-832.

56. Voloshin O, Gocheva Y, Gutnick M, Movshovich N, Bakhrat A, Baranes-Bachar K, Bar-Zvi D, Parvari R, Gheber L, Raveh D: Tubulin chaperone E binds microtubules and proteasomes and protects against misfolded protein stress. Cell Mol Life Sci 2010, 67:2025-2038.

57. Tokito MK, Holzbaur EL: The genomic structure of DCTN1, a candidate gene for limb-girdle muscular dystrophy (LGMD2B). Biochim Biophys Acta 1998, 1442:432-436

58. Dixit R, Levy JR, Tokito M, Ligon LA, Holzbaur ELF: Regulation of Dynactin through the Differential Expression of p150Glued Isoforms. J Biol Chem 2008, 283:33611-33619.

59. Honnappa S, Okhrimenko O, Jaussi R, Jawhari H, Jelesarov I, Winkler FK, Steinmetz MO: Key interaction modes of dynamic + TIP networks. Mol Cell 2006, 23:663-671.

60. Hayashi I, Wilde A, Mal TK, Ikura M: Structural Basis for the Activation of Microtubule Assembly by the EB1 and p150Glued Complex. Mol Cell 2005, 19:449-460.

61. Hayashi I, Plevin MJ, Ikura M: CLIP170 autoinhibition mimics intermolecular interactions with p150Glued or EB1. Nat Struct Mol Biol 2007, 14:980-981.

62. McGrail M, Gepner J, Silvanovich A, Ludmann S, Serr M, Hays TS: Regulation of cytoplasmic dynein function in vivo by the Drosophila Glued complex. J Cell Biol 1995, 131:411-425.

63. Puls I, Jonnakuty C, LaMonte BH, Holzbaur EL, Tokito M, Mann E, Floeter MK, Bidus K, Drayna D, Oh SJ, Brown RH, Ludlow CL, Fischbeck KH: Mutant dynactin in motor neuron disease. Nat Genet 2003, 33:455-456.

64. Farrer MJ, Hulihan MM, Kachergus JM, Dachsel JC, Stoessl AJ, Grantier LL, Calne S, Calne DB, Lechevalier B, Chapon F, Tsuboi Y, Yamada T, Gutmann L, Elibol B, Bhatia KP, Wider C, Vilarino-Guell C, Ross OA, Brown LA, Castanedes-Casey M, Dickson DW, Wszolek ZK: DCTN1 mutations in Perry syndrome. Nat Genet 2009, 41:163-165.

65. Ahmed S, Sun S, Siglin AE, Polenova T, Williams JC: Disease-Associated Mutations in the p150Glued Subunit Destabilize the CAP-gly Domain. Biochemistry 2010, 49:5083-5085.

66. Yue L, Lu S, Garces J, Jin T, Li J: Protein Kinase C-regulated Dynamitin-Macrophage-enriched Myristoylated Alanine-Rice C Kinase Substrate Interaction Is Involved in Macrophage Cell Spreading. J Biol Chem 2000, 275:23948-23956.

67. Maier KC, Godfrey JE, Echeverri CJ, Cheong FKY, Schroer TA: Dynamitin Mutagenesis Reveals Protein-Protein Interactions Important for Dynactin Structure. Traffic 2008, 9:481-491.

68. Garces JA, Clark IB, Meyer DI, Vallee RB: Interaction of the p62 subunit of dynactin with Arp1 and the cortical actin cytoskeleton. Curr Biol 1999, 9:1497-1500.

69. Karki S, Tokito MK, Holzbaur ELF: A Dynactin Subunit with a Highly Conserved Cysteine-rich Motif Interacts Directly with Arp1. J Biol Chem 2000, 275:4834-4839.

70. Eisenhaber B, Chumak N, Eisenhaber F, Hauser M-T: The ring between ring fingers (RBR) protein family. Genome Biol 2007, 8:209.

71. Kadrmas JL, Beckerle MC: The LIM domain: from the cytoskeleton to the nucleus. Nat Rev Mol Cell Biol 2004, 5:920-931.

72. Krishna SS, Majumdar I, Grishin NV: Structural classification of zinc fingers. Nucleic Acids Res 2003, 31:532-550.

73. Schmeichel KL, Beckerle MC: Molecular dissection of a LIM domain. Mol Biol Cell 1997, 8:219-230.

74. Cole C, Barber JD, Barton GJ: The Jpred 3 secondary structure prediction server. Nucleic Acids Res 2008, 36:W197-W201.

75. Scannell DR, Frank AC, Conant GC, Byrne KP, Woolfit M, Wolfe KH: Independent sorting-out of thousands of duplicated gene pairs in two yeast species descended from a whole-genome duplication. Proc Natl Acad Sci U S A 2007, 104:8397-8402.

76. Zhang J, Yao X, Fischer L, Abenza JF, Peñalva MA, Xiang X: The p25 subunit of the dynactin complex is required for dynein-early endosome interaction. J Cell Biol 2011, 193:1245-1255.

77. Lees-Miller JP, Helfman DM, Schroer TA: A vertebrate actin-related protein is a component of a multisubunit complex involved in microtubulebased vesicle motility. Nature 1992, 359:244-246.

78. Clark SW, Meyer DI: Centractin is an actin homologue associated with the centrosome. Nature 1992, 359:246-250. 
79. Clark SW, Staub O, Clark IB, Holzbaur EL, Paschal BM, Vallee RB, Meyer DI: Beta-centractin: characterization and distribution of a new member of the centractin family of actin-related proteins. Mol Biol Cell 1994, 5:1301-1310.

80. Van de Peer $Y$, Maere $S$, Meyer $A: 2 R$ or not $2 R$ is not the question anymore. Nat Rev Genet 2010, 11:166.

81. Kozielski F, Riaz T, DeBonis S, Koehler CJ, Kroening M, Panse I, Strozynski M, Donaldson IM, Thiede B: Proteome analysis of microtubule-associated proteins and their interacting partners from mammalian brain. Amino Acids 2010, 41:363-385.

82. Poch $\mathrm{O}$, Winsor B: Who's Who among the Saccharomyces cerevisiae Actin-Related Proteins? A Classification and Nomenclature Proposal for a Large Family. Yeast 1998, 13:1053-1058.

83. Muller J, Oma Y, Vallar L, Friederich E, Poch O, Winsor B: Sequence and Comparative Genomic Analysis of Actin-related Proteins. Mol Biol Cell 2005, 16:5736-5748.

84. Simpson AGB, Inagaki $Y$, Roger AJ: Comprehensive multigene phylogenies of excavate protists reveal the evolutionary positions of "primitive" eukaryotes. Mol Biol Evol 2006, 23:615-625.

85. Parfrey LW, Grant J, Tekle YI, Lasek-Nesselquist E, Morrison HG, Sogin ML, Patterson DJ, Katz LA: Broadly sampled multigene analyses yield a well-resolved eukaryotic tree of life. Syst Biol 2010, 59:518-533.

86. Burki F, Shalchian-Tabrizi K, Minge M, Skjaeveland A, Nikolaev SI, Jakobsen KS, Pawlowski J: Phylogenomics reshuffles the eukaryotic supergroups. PLoS One 2007, 2:e790.

87. Nozaki H, Maruyama S, Matsuzaki M, Nakada T, Kato S, Misawa K: Phylogenetic positions of Glaucophyta, green plants (Archaeplastida) and Haptophyta (Chromalveolata) as deduced from slowly evolving nuclear genes. Mol Phylogenet Evol 2009, 53:872-880.

88. Keeling PJ: Chromalveolates and the evolution of plastids by secondary endosymbiosis. J Eukaryot Microbiol 2009, 56:1-8.

89. Hackett JD, Yoon HS, Li S, Reyes-Prieto A, Rümmele SE, Bhattacharya D: Phylogenomic analysis supports the monophyly of cryptophytes and haptophytes and the association of rhizaria with chromalveolates. $\mathrm{Mol}$ Biol Evol 2007, 24:1702-1713.

90. Burki F, Shalchian-Tabrizi K, Pawlowski J: Phylogenomics reveals a new "megagroup" including most photosynthetic eukaryotes. Biol Lett 2008, 4:366-369.

91. Reeb VC, Peglar MT, Yoon HS, Bai JR, Wu M, Shiu P, Grafenberg JL, ReyesPrieto A, Rümmele SE, Gross J, Bhattacharya D: Interrelationships of chromalveolates within a broadly sampled tree of photosynthetic protists. Mol Phylogenet Evol 2009, 53:202-211.

92. Hampl V, Hug L, Leigh JW, Dacks JB, Lang BF, Simpson AGB, Roger AJ: Phylogenomic analyses support the monophyly of Excavata and resolve relationships among eukaryotic "supergroups". Proc Natl Acad Sci U S A 2009, 106:3859-3864.

93. Derelle R, Lang BF: Rooting the eukaryotic tree with mitochondrial and bacterial proteins. Mol Biol Evol 2012, 29:1277-1289.

94. Keeling PJ, Burger G, Durnford DG, Lang BF, Lee RW, Pearlman RE, Roger AJ, Gray MW: The tree of eukaryotes. Trends Ecol Evol 2005, 20:670-676.

95. Stechmann A, Cavalier-Smith T: Phylogenetic analysis of eukaryotes using heat-shock protein Hsp90. J Mol Evol 2003, 57:408-419.

96. Rogozin IB, Basu MK, Csürös M, Koonin EV: Analysis of rare genomic changes does not support the unikont-bikont phylogeny and suggests cyanobacterial symbiosis as the point of primary radiation of eukaryotes. Genome Biol Evol 2009, 1:99-113.

97. Koonin EV: The origin and early evolution of eukaryotes in the light of phylogenomics. Genome Biol 2010, 11:209.

98. Eme L, Trilles A, Moreira D, Brochier-Armanet C: The phylogenomic analysis of the anaphase promoting complex and its targets points to complex and modern-like control of the cell cycle in the last common ancestor of eukaryotes. BMC Evol Biol 2011, 11:265.

99. Moparthi VK, Hägerhäll C: The evolution of respiratory chain complex I from a smaller last common ancestor consisting of 11 protein subunits. J Mol Evol 2011, 72:484-497.

100. Tokito MK, Howland DS, Lee VM, Holzbaur EL: Functionally distinct isoforms of dynactin are expressed in human neurons. Mol Biol Cell 1996 , 7:1167-1180.

101. Zhapparova ON, Bryantseva SA, Dergunova LV, Raevskaya NM, Burakov AV, Bantysh OB, Shanina NA, Nadezhdina ES: Dynactin subunit p150Glued isoforms notable for differential interaction with microtubules. Traffic 2009, 10:1635-1646.
102. Vaughan PS, Miura $P$, Henderson M, Byrne $B$, Vaughan KT: A role for regulated binding of $\mathrm{p} 150$ (Glued) to microtubule plus ends in organelle transport. J Cell Biol 2002, 158:305-319.

103. Parisi G, Fornasari MS, Echave J: Dynactins p25 and p27 are predicted to adopt the LbetaH fold. FEBS Lett 2004, 562:1-4.

104. Yeh E, Skibbens RV, Cheng JW, Salmon ED, Bloom K: Spindle dynamics and cell cycle regulation of dynein in the budding yeast, Saccharomyces cerevisiae. J Cell Biol 1995, 130:687-700.

105. Moore JK, Li J, Cooper JA: Dynactin function in mitotic spindle positioning. Traffic 2008, 9:510-527.

106. Kurtzman CP: Phylogeny of the ascomycetous yeasts and the renaming of Pichia anomala to Wickerhamomyces anomalus. Antonie Van Leeuwenhoek 2011, 99:13-23.

107. Curtin CD, Borneman AR, Chambers PJ, Pretorius IS: De-Novo Assembly and Analysis of the Heterozygous Triploid Genome of the Wine Spoilage Yeast Dekkera bruxellensis AWRI1499. PLoS One 2012, 7:e33840.

108. Odronitz F, Kollmar M: Pfarao: a web application for protein family analysis customized for cytoskeletal and motor proteins (CyMoBase). BMC Genomics 2006, 7:300.

109. Hatje K, Keller O, Hammesfahr B, Pillmann H, Waack S, Kollmar M: Cross-species protein sequence and gene structure prediction with finetuned Webscipio 2.0 and Scipio. BMC Res Notes 2011, 4:265.

110. Chenna R, Sugawara H, Koike T, Lopez R, Gibson TJ, Higgins DG, Thompson JD: Multiple sequence alignment with the Clustal series of programs. Nucleic Acids Res 2003, 31:3497-3500.

111. Talavera G, Castresana J: Improvement of phylogenies after removing divergent and ambiguously aligned blocks from protein sequence alignments. Syst Biol 2007, 56:564-577.

112. Campanella JJ, Bitincka L, Smalley J: MatGAT: an application that generates similarity/identity matrices using protein or DNA sequences. BMC Bioinforma 2003, 4:29.

113. Darriba D, Taboada GL, Doallo R, Posada D: ProtTest 3: fast selection of best-fit models of protein evolution. Bioinformatics 2011, 27:1164-1165.

114. Le SQ, Gascuel O: An improved general amino acid replacement matrix. Mol Biol Evol 2008, 25:1307-1320.

115. Jones DT, Taylor WR, Thornton JM: The rapid generation of mutation data matrices from protein sequences. Comput App/ Biosci 1992, 8:275-282.

116. Stamatakis $A$, Hoover $P$, Rougemont J: A rapid bootstrap algorithm for the RAxML Web servers. Syst Bio/ 2008, 57:758-771.

117. Ronquist F, Huelsenbeck JP: MrBayes 3: Bayesian phylogenetic inference under mixed models. Bioinformatics 2003, 19:1572-1574.

118. Altekar G, Dwarkadas S, Huelsenbeck JP, Ronquist F: Parallel Metropolis coupled Markov chain Monte Carlo for Bayesian phylogenetic inference. Bioinformatics 2004, 20:407-415.

119. Whelan S, Goldman N: A general empirical model of protein evolution derived from multiple protein families using a maximum-likelihood approach. Mol Biol Evol 2001, 18:691-699.

120. Pillmann H, Hatje K, Odronitz F, Hammesfahr B, Kollmar M: Predicting mutually exclusive spliced exons based on exon length, splice site and reading frame conservation, and exon sequence homology. BMC Bioinforma 2011, 12:270.

121. Schafer DA, Korshunova YO, Schroer TA, Cooper JA: Differential localization and sequence analysis of capping protein beta-subunit isoforms of vertebrates. J Cell Biol 1994, 127:453-465

doi:10.1186/1471-2148-12-95

Cite this article as: Hammesfahr and Kollmar: Evolution of the eukaryotic dynactin complex, the activator of cytoplasmic dynein. BMC Evolutionary Biology 2012 12:95. 